\title{
Ball Bearing Model Performance on Various Sized Rotors with and without Centrifugal and Gyroscopic Forces
}

\author{
Emil Kurvinen ${ }^{\mathrm{a}, *}$, Jussi Sopanen ${ }^{\mathrm{a}}$, Aki Mikkola ${ }^{\mathrm{a}}$ \\ ${ }^{a}$ Lappeenranta University of Technology, Department of Mechanical Engineering, \\ P.O. Box 20, FI-53851 Lappeenranta, Finland
}

\section{Abstract}

Bearing performance significantly affects the dynamic behaviors and estimated working life of a rotating system. A common bearing type is the ball bearing, which has been under investigation in numerous published studies. The complexity of the ball bearing models described in the literature varies. Naturally, model complexity is related to computational burden. In particular, the inclusion of centrifugal forces and gyroscopic moments significantly increases the system degrees of freedom and lengthens solution time. On the other hand, for low or moderate rotating speeds, these effects can be neglected without significant loss of accuracy. The objective of this paper is to present guidelines for the appropriate selection of a suitable bearing model for three case studies. To this end, two ball bearing models were implemented. One considers high-speed forces, and the other neglects them. Both models were used to study a three structures, and the simulation results were

\footnotetext{
*Corresponding author. Mobile +358 50569 5969, Fax +358 56212499

Email addresses: emil.kurvinen@lut.fi (Emil Kurvinen ), jussi.sopanen@lut.fi (Jussi Sopanen), aki.mikkola@lut.fi (Aki Mikkola)
} 
compared. The bearing behavior is studied at different shaft rotation speeds and the simulation results are used to determine when the model containing the centrifugal and gyroscopic forces should be used.

Keywords: Ball bearing, centrifugal forces, dynamical analysis, gyroscopic moment, inertia effect

\section{NOMENCLATURE}

$\begin{array}{ll}\begin{array}{ll}\text { Latin } \\ c_{\mathrm{d}}\end{array} & \text { Clearance } \\ d & \text { Ball diameter } \\ d_{\mathrm{m}} & \text { Pitch diameter } \\ d_{\mathrm{s}} & \text { Bore diameter } \\ d_{\mathrm{m}}^{\prime} & \text { Pitch diameter under loading } \\ D & \text { Diameter } \\ e & \text { Eccentricity } \\ E^{\prime} & \text { Effective modulus of elasticity } \\ E & \text { Modulus of elasticity } \\ f & \text { Conformity ratio } \\ F & \text { Force } \\ I_{\mathrm{p}} & \text { Polar moment of inertia } \\ \mathrm{j} & \text { Number of ball } \\ K_{\text {con }} & \text { Contact stiffness coefficient } \\ \tilde{k}_{\mathrm{e}} & \text { Approximated ellipticity parameter } \\ L & \text { Distance (Figure 6) }\end{array}$




\begin{tabular}{|c|c|}
\hline $\mathrm{m}$ & Mass of the ball \\
\hline $\mathrm{n}$ & Number of balls \\
\hline$M_{\mathrm{g}}$ & Gyroscopic moment \\
\hline$r$ & Radius \\
\hline$R$ & Curvature sum \\
\hline$R_{\mathrm{d}}$ & Curvature difference \\
\hline$R_{\mathrm{x}}$ & Effective radii of curvature in $x$-plane \\
\hline$R_{\mathrm{y}}$ & Effective radii of curvature in y-plane \\
\hline$T$ & Moment \\
\hline \multicolumn{2}{|c|}{ Greek } \\
\hline$\alpha$ & Contact angle (improved model) \\
\hline$\beta$ & Attitude angle (azimuth angle) (simplified model) \\
\hline$\gamma$ & Misalignment \\
\hline$\delta$ & Deformation \\
\hline$\tilde{\zeta}$ & Second ellipticity integral \\
\hline$\theta$ & Angle between the shaft centerline and ball rotational axis \\
\hline$\tilde{\xi}$ & First ellipticity integral \\
\hline$\lambda$ & Race control \\
\hline$\nu$ & Poisson's ratio \\
\hline$\phi$ & Contact angle (simplified model) \\
\hline$\psi$ & Angular location (improved model) \\
\hline$\omega$ & Rotation speed \\
\hline
\end{tabular}




\begin{tabular}{|c|c|}
\hline \multicolumn{2}{|c|}{ Subscripts } \\
\hline $\mathrm{a} y$ & Solid a, $y$-direction \\
\hline $\mathrm{a} x$ & Solid a, $x$-direction \\
\hline B & Ball \\
\hline by & Solid b, $y$-direction \\
\hline $\mathrm{b} x$ & Solid b, $x$-direction \\
\hline c & Centrifugal \\
\hline f & Initial contact angle \\
\hline $\mathrm{h}$ & housing \\
\hline $\mathrm{i} /$ in & Inner race \\
\hline j & $\mathrm{j}$ th ball \\
\hline o/out & Outer race \\
\hline $\mathrm{s}$ & Shaft \\
\hline$x$ & Axis $x$-direction \\
\hline$y$ & Axis $y$-direction \\
\hline$z$ & Axis $z$-direction \\
\hline \multicolumn{2}{|c|}{ Superscripts } \\
\hline in & Inner \\
\hline out & Outer \\
\hline $\mathrm{r}$ & Radial \\
\hline $\mathrm{t}$ & Axial \\
\hline tot & Total \\
\hline
\end{tabular}




\section{Introduction}

Bearings have an important role in every rotating machinery systems. One of the commonly used bearing types is a ball bearing, which is under investigation in this paper. Modeling the dynamical behavior of a ball bearing includes several simultaneous contacts between the bearing components that make the accurate simulation of a ball bearing challenging and computationally heavy. The modeling and simulation provide accurate information of the dynamic performance of systems that contain ball bearings. However, depending on the complexity of the model, the computation time varies. It is possible to simulate ball bearings with excellent accuracy but then there is a need for more input parameters, including those that are not widely available.

The basic concept in ball bearings is that the balls rotate inside two steel rings (races) and the balls are kept separate with a cage. The design varies depending on the specific type of bearing but the basic concept is the same in every ball bearing.

Jones [1] presented a general theory for modeling an elastically constrained ball and radial roller bearing. The model considers five degrees of freedom of the inner ring and includes gyroscopic moments and centrifugal forces of the balls. The proposed model was verified later by Harris [2], Hamrock and Dowson [3] who generalized the proposed theory. The proposed model did not include any geometrical imperfections, which led to numerous studies considering the effects of different kinds of defects in the bearing. There is also a general trend that the earlier models considered fever degrees

of freedom than more recently proposed models when studying the effects of 
imperfections to the dynamical behavior of rotor bearing systems.

Tandon and Choudhury [4] presented a thorough review for detecting defects in rolling element bearings. They classified bearing defects into two categories, distributed and localized. The defects caused by manufacturing error, improper installation or abrasive wear are called distributed defects; those include surface roughness, waviness, misaligned races and off-size rolling elements. The defects caused by fatigue are called localized defects; those include cracks, pits and spalls on the rolling surfaces.

Aktürk et al. $[5,6]$ proposed a three-degree-of-freedom ball bearing model where the effects of waviness, axial preload, and the number of balls were studied.

Jang and Jeong [7] proposed a five-degrees-of-freedom model for a rigid rotor that is supported at least with two bearings. The model is used to study the waviness in races and balls. The results are verified with prior researchers. Further, Jang and Jeong [8] included the gyroscopic moment and centrifugal force of the balls in the model and studied the stiffness, contact force, displacement and vibration frequencies behavior with different waviness order in each bearing and validated the results with prior researchers. Jang and Jeong [9] studied the effect of different waviness order in the model that contains a pair of bearings and also the eccentricity of the center of mass of the rotor and bearing span center. The inertia forces were found to have a major effect when determining the bearing vibration frequencies.

Sopanen and Mikkola [10] presented a six-degrees-of-freedom ball bearing model theory where the sixth degree of freedom is the friction torque around the rotational axis. The model considers waviness in the inner and outer races 
as well as the localized defects in the races. In the second part, Sopanen and Mikkola [11] investigated the clearance variation in the dynamical behavior, which was found to have a major effect. The low-order waviness generates vibrations at the frequencies at waviness order multiplied with rotation speed. They also concluded that when the waviness is near the number of balls, the vibrations occur near the ball passage frequencies in the races. The localized defects found to generate frequencies at the bearing defect frequency. The results were verified with the results available in the literature. The model does not consider the centrifugal force and gyroscopic moment.

Changqing and Qinqyu [12] propose a model that has five degrees of freedom and considers both centrifugal force and gyroscopic moment. They studied a rotor bearing system under internal clearance, axial preload, radial load and waviness. The first three were found to have a significant role in systems stability, and the outer race waviness was found to have the most effect compared to inner race and ball waviness. Changqing et al. [13] improved the proposed model and studied the axial preload effect on the ball bearings dynamical behavior. In the study, it was concluded that the unstable periodic solution of a balanced rotor can be avoided with the proper axial preload.

Recently, Nakhaeinejad and Bryant [14] proposed a model of rolling element bearings by using graphical vector bond method. The model consists of nine balls and two rings. The model includes centrifugal force and gyroscopic moment, contact elastic deflections and forces, contact slip, contact separation and defects on races. The model was verified with a test machine and by using the available solutions in the literature. However, the planar 
model does not consider the axial preload and its effect on the stiffness of the bearing. Xu et al. [15] studied a crankshaft mechanism where the revolute joint has been modeled as a ball bearing. The balls are modeled as individual parts. In the research, the clearance effect and number of balls influencing the load that individual ball is having are studied. Xu et al. concluded that by having more balls, the load per individual part is decreased and the displacement is smaller compared to bearings that have fewer balls.

In conclusion, the research done in developing the ball bearing model can be categorized into two main fields: the ones which consider dynamical forces due to high rotation speed and the simplified ones that do not. The research done earlier usually does not consider the dynamical forces and the models developed more recently do. It is found that the ball bearing that is modeled using five degrees of freedom can be used to simulate the dynamical behavior under imperfections (due manufacturing and installation) accurately. Friction force can be also added to the model, if the studied application has a special need for that, (e.g., studying the touchdown bearing behavior when magnetic bearings fails). However, when considering several degrees of freedom and inertia forces, solving the model becomes computationally heavy to solve. Liew et al. [16] studied the effect of a two and a five-degrees-of-freedom bearing model with inertia and without. In the study, it was concluded that the five-degrees-of-freedom model with inertia forces needs 10 times more time to solve than without the inertia forces.

The objective of this research is to improve the six-degrees-of-freedom ball bearing proposed by Sopanen and Mikkola [10,11] by including the centrifugal force and gyroscopic moment to the model. As suggested by Kurvinen et 
al. [17] the models should be implemented into different structures with various sized bearings to The proposed models are utilized in the simulation of rotor dynamics in the simulation of three industrial applications, a high-speed electric motor (turbo blower), a high-speed machine and a high-speed generator. The applications are suited with a pair of different sized bearings; $70 \mathrm{~mm}, 55 \mathrm{~mm}$ and $25 \mathrm{~mm}$, respectively. The results obtained using the bearing models with and without ball inertia effects are compared and the justification for the inclusion of computationally expensive centrifugal force and gyroscopic moment effects is discussed. Finally, the results are used to determine at what rotation speed inertia forces can be neglected and at what speed they are necessary to consider.

\section{Methods}

The simplified model is based on the relative displacements and velocities between the inner and outer races. Displacements and velocities can be calculated by creating a geometrical relationship. Accordingly, the relationship between the force-deflection can be expressed. The model has five degrees of freedom: three translational and two rotational degrees of freedom. The model includes non-linear Hertzian contact deformation. The geometry and material parameters are given as input and the bearing forces and torques are solved. The simplified model does not consider high-speed forces.

The refined model is based on the force equilibrium between the external applied load and the internal load within the bearing. Rotation dependent forces, such as centrifugal force and gyroscopic moment of the ball are included. In the equilibrium condition, the deformation of the ball bearing is 
solved.

The ball bearing contains several parts including the cage that holds the balls in a fixed distance from the inner and outer race. The races connects the bearing to the shaft and housing. Each part should be considered in details when creating an accurate mathematical model. However, the model should be computationally efficient and therefore the following general assumptions are made to decrease the degrees of freedom in the models:

1. Friction torque is neglected.

2. The effect of elastohydrodynamic film is neglected.

3. The defects and form error of the unit are neglected.

The friction torque and the effects of the hydrodynamic film are neglected in order to decrease model complexity. The effects of those additional parameters are assumed to have similar effects on both models. The defects and form error of the bearing are not included and they are assumed to be perfectly manufactured (i.e., the balls are round and races are circular.)

\subsection{Ball bearing dynamic model without centrifugal and gyroscopic forces}

The theory of modeling the deep-groove ball bearing is presented in detail in [10]. The modeling theory is briefly reviewed in this paper in order to provide a complete presentation. In this study, the ball bearing model without centrifugal and gyroscopic forces is called the "simplified model".

\subsubsection{Elliptical contact conjunction}

The elliptical contact conjunction is used to determine the contact stiffness between the ball and races. In ball bearings, the ball and race are two 
solids that have a different radii of curvature in two directions. Figure 1 shows the contact geometry and the normal force, $F$, applied to two solids. The radius of contact conjunction is defined to be positive when the surface is convex and negative when concave.

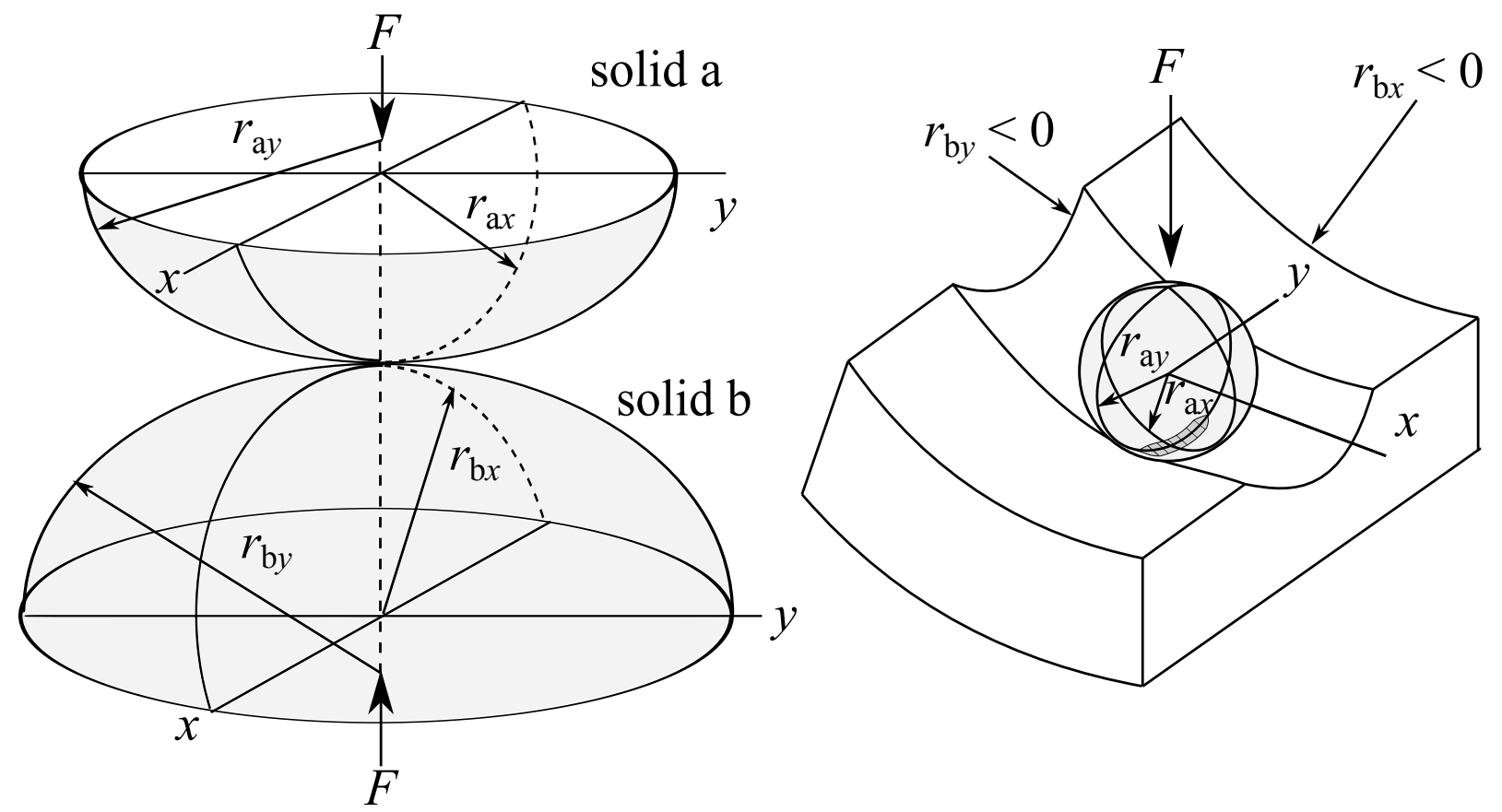

Figure 1: Elliptical contact conjuctions

The curvature sum, $R$, and curvature difference, $R_{d}$, that are used to define the contact deformation are as 


$$
\begin{aligned}
& \frac{1}{R}=\frac{1}{R_{x}}+\frac{1}{R_{y}} \\
& R_{\mathrm{d}}=R\left(\frac{1}{R_{x}}-\frac{1}{R_{y}}\right),
\end{aligned}
$$

where

$$
\frac{1}{R_{x}}=\frac{1}{r_{\mathrm{a} x}}+\frac{1}{r_{\mathrm{b} x}}, \frac{1}{R_{y}}=\frac{1}{r_{\mathrm{a} y}}+\frac{1}{r_{\mathrm{b} y}}
$$

where $R_{x}$ and $R_{y}$ represent the effective radii of curvature in the $x$ and $y$ planes. Subscripts $\mathrm{a} x, \mathrm{a} y, \mathrm{~b} x$ and $\mathrm{b} y$ refer to the radii in solids a and $\mathrm{b}$ in $x$ - and $y$-directions, respectively. When the normal force is applied to the solids, the contact area is elliptical. The contact stiffness coefficient, $K_{\text {con }}$, for the elliptical contact conjunction can be calculated as

$$
\begin{aligned}
& K_{\text {con }}=\pi \tilde{k}_{\mathrm{e}} E^{\prime} \sqrt{\frac{R \tilde{\xi}}{4.5 \tilde{\zeta}^{3}}}, \\
& \text { where } \\
& \tilde{k}_{\mathrm{e}}=1.0339\left(\frac{R_{y}}{R_{x}}\right)^{0.6360,} \\
& E^{\prime}=\frac{2}{\frac{1-\nu_{\mathrm{a}}^{2}}{E_{\mathrm{a}}}+\frac{1-\nu_{\mathrm{b}}^{2}}{E_{\mathrm{b}}}}, \\
& \tilde{\xi}=1.003+0.5968\left(\frac{R_{y}}{R_{x}}\right), \\
& \tilde{\zeta}=1.5277+0.6023 \ln \left(\frac{R_{y}}{R_{x}}\right) .
\end{aligned}
$$

where $\tilde{k}_{\mathrm{e}}$ is the approximated ellipticity parameter, $E^{\prime}$ is the effective modulus of elasticity, $\nu$ is the Poisson's ratio, $E$ is the material modulus of elasticity and subscripts a and b refer to solids that are in contact. Parameters $\tilde{\xi}$ and $\tilde{\zeta}$ are the approximation formulae for first and second elliptical integrals [3]. 


\subsubsection{Ball bearing geometry, total stiffness coefficient and displacements}

The geometry of a deep groove ball bearing is shown in Fig. 2, where $d$ is the ball diameter, $r_{\text {out }}$ the outer groove radius, $r_{\text {in }}$ is the inner groove radius, $c_{\mathrm{d}}$ is the diametral clearance, $D_{\mathrm{h}}$ is the bearing housing diameter, $d_{\mathrm{m}}$ is the pitch diameter, $d_{\mathrm{s}}$ is the bore diameter, $D_{\mathrm{i}}$ is the inner raceway diameter, and $D_{\mathrm{o}}$ is the outer raceway diameter, respectively.
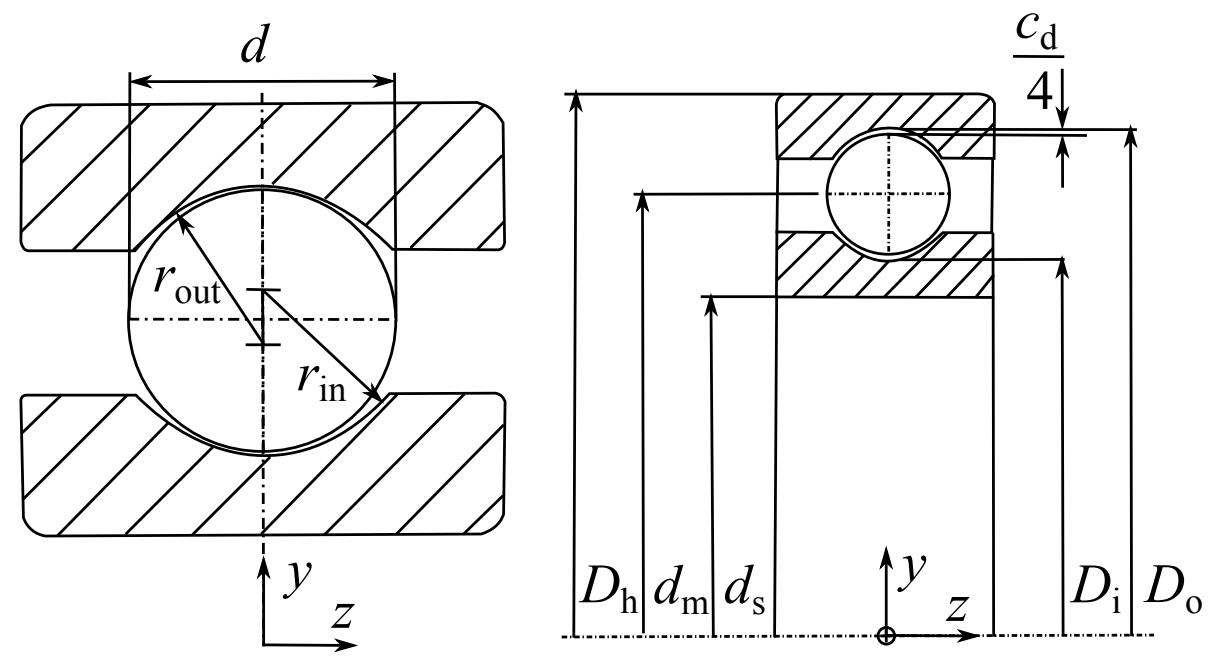

Figure 2: Main dimensions of a ball bearing

The relation between contact stiffness and deformation, $\delta_{0}$, for a single ball is expressed as [3]

$$
\delta_{0}=\left(\frac{F}{K_{\mathrm{con}}}\right)^{2 / 3},
$$

where $F$ is the normal load. The total stiffness of a single ball bearing involves the inner, $K_{\mathrm{con}}^{\mathrm{in}}$, and the outer ring, $K_{\mathrm{con}}^{\mathrm{out}}$, contact stiffness and that is expressed as [3] 


$$
K_{\mathrm{con}}^{\mathrm{tot}}=\frac{1}{\left(\left(\frac{1}{K_{\mathrm{con}}^{\text {in }}}\right)^{2 / 3}+\left(\frac{1}{K_{\mathrm{con}}^{\text {out }}}\right)^{2 / 3}\right)^{3 / 2}} .
$$

\subsubsection{Ball bearing forces and moments in the complete ball bearing}

Ball bearing forces and moments are calculated from the relative displacements between the rings. Figure 3 shows the layout for each ball and the relative displacements between the inner and outer ring.
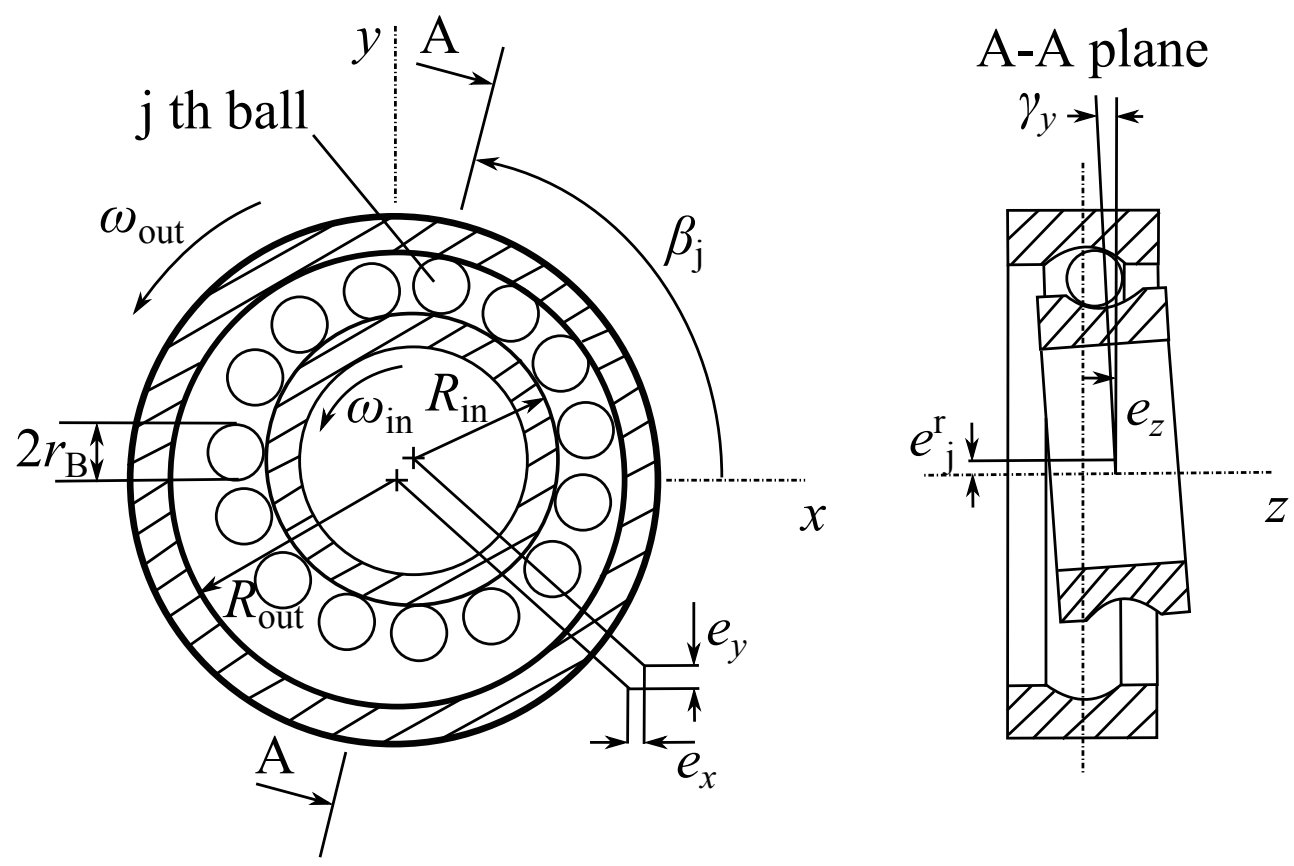

Figure 3: Axial and transverse cross-section in the A-A plane of illustrative structure of a ball bearing

Radial, $e_{\mathrm{j}}^{\mathrm{r}}$, and axial, $e_{\mathrm{j}}^{\mathrm{t}}$, displacements in of ball $\mathrm{j}$ can be stated as 


$$
\begin{aligned}
& e_{\mathrm{j}}^{\mathrm{r}}=e_{x} \cos \beta_{\mathrm{j}}+e_{y} \sin \beta_{\mathrm{j}} \\
& e_{\mathrm{j}}^{\mathrm{t}}=e_{z}-\left(-\gamma_{x} \sin \beta_{\mathrm{j}}+\gamma_{y} \cos \beta_{\mathrm{j}}\right)\left(R_{\mathrm{in}}+r_{\mathrm{in}}\right)
\end{aligned}
$$

where $e_{x}, e_{y}, e_{z}$ are the given relative displacements in $x, y, z$ directions, $\beta_{\mathrm{j}}$ is the attitude angle (azimuth angle) of ball $\mathrm{j}$ and $\gamma_{x}$ and $\gamma_{y}$ are the angular misalignments of the inner race about $x$ and $y$ axes. The attitude angle can be calculated for jth ball as follow

$$
\beta_{\mathrm{j}}=\frac{2 \pi(\mathrm{j}-1)}{n},
$$

where $n$ is the number of balls in the bearing [3]. The contact angle for ball $\mathrm{j}, \phi_{\mathrm{j}}$, is depicted in Fig. 4.

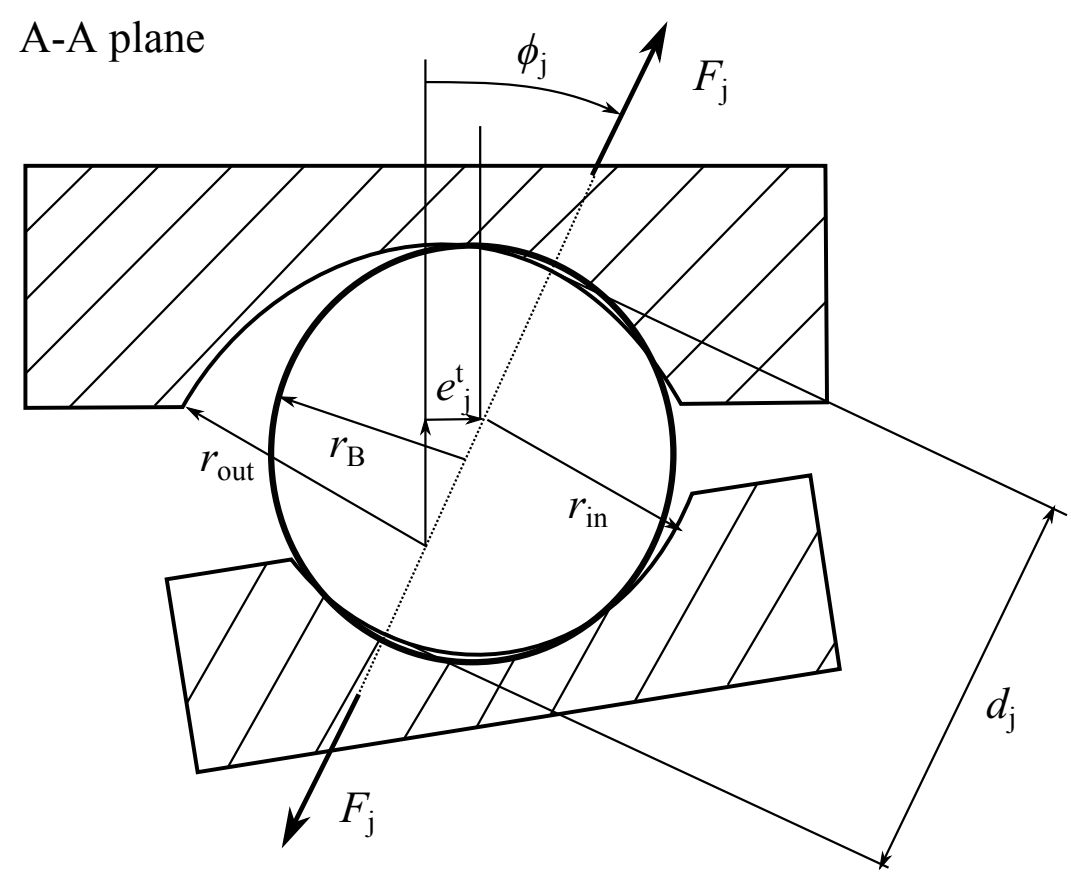

Figure 4: Cross-section of a ball bearing in the A-A plane 
The contact angle, $\phi_{\mathrm{j}}$, for ball $\mathrm{j}$ can be calculated as

$$
\phi_{\mathrm{j}}=\tan ^{-1}\left(\frac{e_{\mathrm{j}}^{\mathrm{t}}}{R_{\mathrm{in}}+r_{\mathrm{in}}+e_{\mathrm{j}}^{\mathrm{r}}-R_{\text {out }}+r_{\text {out }}}\right) .
$$

The distance between the race surfaces along the line of contact, $d_{\mathrm{j}}$, is then calculated as

$$
d_{\mathrm{j}}=r_{\mathrm{out}}+r_{\mathrm{in}}-\frac{R_{\mathrm{in}}+r_{\mathrm{in}}+e_{\mathrm{j}}^{\mathrm{r}}-R_{\mathrm{out}}+r_{\mathrm{out}}}{\cos \phi_{\mathrm{j}}} .
$$

The total elastic deformation can then be calculated as

$$
\delta_{\mathrm{j}}^{\text {tot }}=2 r_{\mathrm{B}}-d_{\mathrm{j}}
$$

The contact force acting on ball $\mathrm{j}$ can be calculated for each ball as

$$
F_{\mathrm{j}}=K_{\mathrm{con}}^{\mathrm{tot}}\left(\delta_{\mathrm{j}}^{\mathrm{tot}}\right)^{3 / 2}
$$

where $K_{\text {con }}^{\text {tot }}$ is the total stiffness coefficient determined by Eq. 4 [3]. The resultant bearing forces and moments in the shaft can be calculated in respect to the $x, y$ and $z$ directions as

$$
\left.\begin{array}{l}
F_{x}=-\sum_{\mathrm{j}=1}^{n} F_{\mathrm{j}} \cos \phi_{\mathrm{j}} \cos \beta_{\mathrm{j}} \\
F_{y}=-\sum_{\mathrm{j}=1}^{n} F_{\mathrm{j}} \cos \phi_{\mathrm{j}} \sin \beta_{\mathrm{j}} \\
F_{z}=-\sum_{\mathrm{j}=1}^{n} F_{\mathrm{j}} \sin \phi_{\mathrm{j}} \\
T_{x}=-\sum_{\mathrm{j}=1}^{n} F_{\mathrm{j}}\left(R_{\mathrm{in}}+r\right) \sin \phi_{\mathrm{j}} \sin \beta_{\mathrm{j}} \\
T_{y}=-\sum_{\mathrm{j}=1}^{n} F_{\mathrm{j}}\left(R_{\mathrm{in}}+r\right) \sin \phi_{\mathrm{j}}\left(-\cos \beta_{\mathrm{j}}\right)
\end{array}\right\}
$$


It should be noted that in summations in Eq. 10 only the force components where compression exists between the races and ball are included, (i.e., $\left.\delta_{\mathrm{j}}^{\text {tot }}>0\right)$.

\subsection{Ball bearing dynamic model with centrifugal and gyroscopic forces}

In high-speed operations, the contact angles in the inner and outer race contacts are no longer identical. This will have a noticeable effect on the ball bearings dynamic behavior in high-speed. In this study, the ball bearing model with centrifugal and gyroscopic forces is called the "improved model". Figure 5 shows the forces and contact angles within a single ball and the position of the jth ball in the bearing. The ball is subjected to the centrifugal force, $F_{\mathrm{c}}$, and the contact forces on the inner and outer races, $F_{\mathrm{i}}$ and $F_{\mathrm{o}}$. In addition, a gyroscopic moment, $M_{\mathrm{g}}$, and counter friction forces are affecting the ball deformation. The force equilibrium for a single ball can be calculated when the contact angles are known in the inner and outer races $\alpha_{\mathrm{i}}$ and $\alpha_{\mathrm{o}}$, respectively. Notation $\lambda$ represent the race control and it has a value of 0 when inner-race control is valid or 1 when outer-race control is valid. 

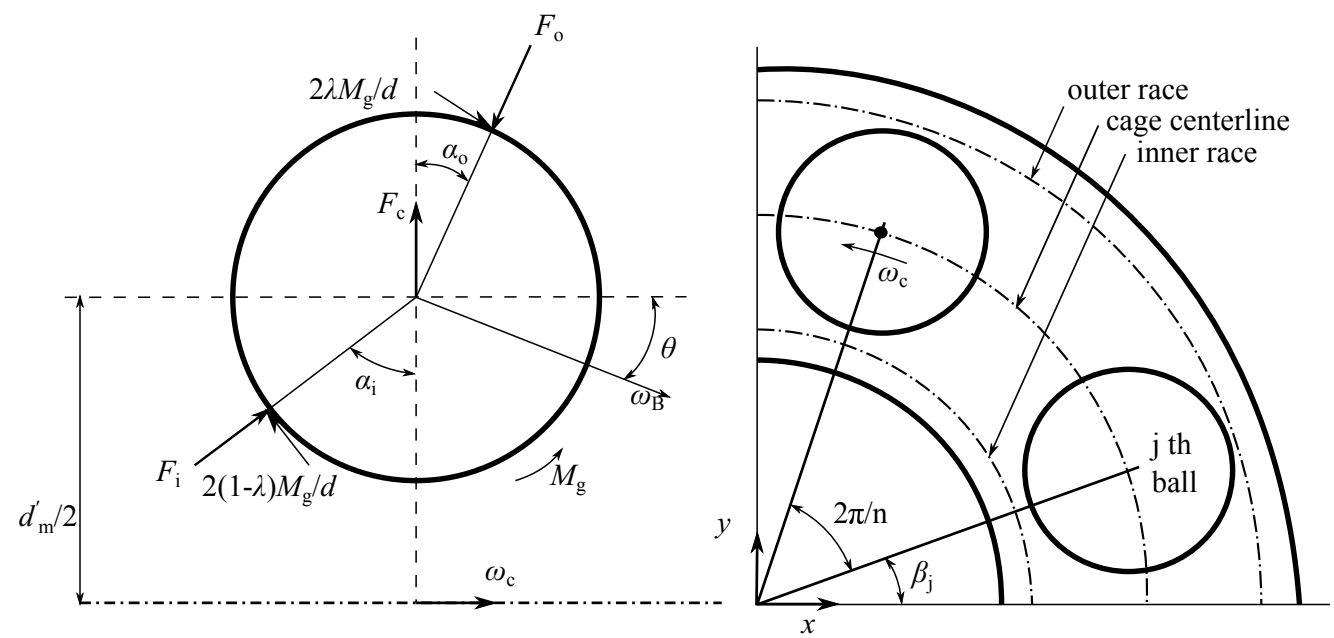

Figure 5: Forces and moments acting on the ball on high-speed operation

In the case of a substantial centrifugal force, the contact angles in the inner and outer race contacts are different. Pitch diameter under loading, $d_{\mathrm{m}}^{\prime}$, can be expressed as a function of nominal pitch diameter $d_{\mathrm{m}}, L_{4}$ distance (Fig. 6), ball diameter $d$, outer race conformity ratio, $f_{\mathrm{o}}$, and initial contact angle, $\alpha_{\mathrm{f}}$, as

$$
d_{\mathrm{m}}^{\prime}=d_{\mathrm{m}}+2 L_{4}-2 d\left(f_{\mathrm{o}}-0.5\right) \cos \alpha_{\mathrm{f}}
$$

The initial contact angle can be calculated from the diametral clearance, $c_{\mathrm{d}}$, ball diameter, $d$, and outer and inner conformity ratio, $f_{\mathrm{o}}$ and $f_{\mathrm{i}}$, as

$$
\alpha_{\mathrm{f}}=\cos ^{-1}\left(1-\frac{c_{\mathrm{d}}}{2 d\left(f_{\mathrm{o}}+f_{\mathrm{i}}-1\right)}\right)
$$

The angle between ball rotational axis and bearing centerline, $\theta$, can be calculated as 


$$
\theta=\tan ^{-1}\left(\frac{d_{\mathrm{m}}^{\prime} \sin \alpha_{\mathrm{o} / \mathrm{i}}}{d_{\mathrm{m}}^{\prime} \cos \alpha_{\mathrm{o} / \mathrm{i}} \pm d}\right)
$$

for the outer-race control the contact angle $\alpha_{\mathrm{o}}$ is used and in the nominator the diameter is added and for the inner-race control contact angle $\alpha_{\mathrm{i}}$ is used and in the nominator the diameter is subtracted. [3]

Figure 6 shows the initial and final (loaded) positions of the ball and inner race curvature center. 


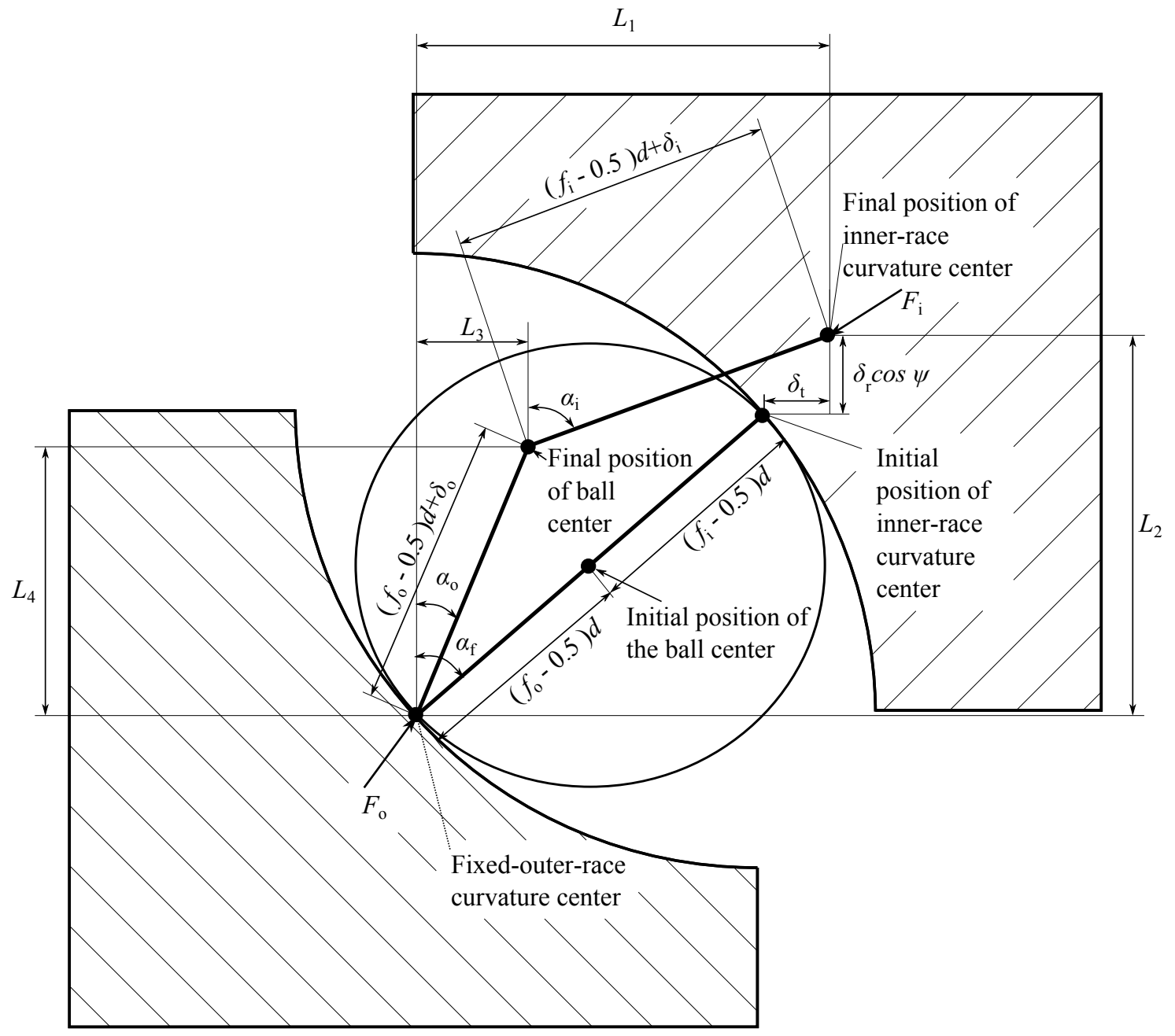

Figure 6: Deflection of the center of ball and curvature races at angular location $\psi[3]$

Based on Fig. 6 the following geometric relation for each ball can be expressed using the Pythagorean theorem as

$$
\begin{aligned}
& L_{4}^{2}+L_{3}^{2}-\left[\left(f_{\mathrm{o}}-0.5\right) d+\delta_{\mathrm{o}}\right]^{2}=0 \\
& \left(L_{2}-L_{4}\right)^{2}+\left(L_{1}-L_{3}\right)^{2}-\left[\left(f_{\mathrm{i}}-0.5\right) d+\delta_{\mathrm{i}}\right]^{2}=0,
\end{aligned}
$$


where $f_{\mathrm{i}}$ and $f_{\mathrm{o}}$ are the inner and outer race conformity ratio that is a measure of the geometrical conformity of the race and the ball in a plane that passes through the center of the bearing and is transverse to the race (shown in Fig. 2). Forces applied to the ball are shown in Fig. 5, and these can be calculated when the total elastic deformation in the inner and outer race contacts and the stiffness coefficients are known as [3]

$$
\begin{aligned}
& F_{\mathrm{o}}=K_{\mathrm{o}} \delta_{\mathrm{o}}^{3 / 2} \\
& F_{\mathrm{i}}=K_{\mathrm{i}} \delta_{\mathrm{i}}^{3 / 2} .
\end{aligned}
$$

The equilibrium condition for each ball can be expressed with the help of Newton's second law as

$$
\begin{aligned}
& F_{\mathrm{o}} \sin \alpha_{\mathrm{o}}-F_{\mathrm{i}} \sin \alpha_{\mathrm{i}}-\frac{2 M_{\mathrm{g}}}{d}\left(\lambda \cos \alpha_{\mathrm{o}}-(1-\lambda) \cos \alpha_{\mathrm{i}}\right)=0 \\
& F_{\mathrm{o}} \cos \alpha_{\mathrm{o}}-F_{\mathrm{i}} \cos \alpha_{\mathrm{i}}+\frac{2 M_{\mathrm{g}}}{d}\left(\lambda \sin \alpha_{\mathrm{o}}-(1-\lambda) \sin \alpha_{\mathrm{i}}\right)-F_{\mathrm{c}}=0,
\end{aligned}
$$

where $\lambda$ has a value of 1 in the case of outer-race control and 0 when innerrace control is applied. The race control theory was proposed by Jones [1], where $\lambda$ is a calculated parameter to define the race in which no slipping occurs. The required torques to spin the ball against the inner and outer race is evaluated. A race, which requires more torque to spin, defines the race control, (i.e., if $\lambda$ is 1 the outer-race control exists). and when $\lambda$ is 0 then the inner race contact exists. In Eq. 17 the centrifugal force can be expressed as

$$
F_{\mathrm{c}}=\frac{1}{2} m d_{\mathrm{m}}^{\prime} \omega_{\mathrm{c}}^{2}
$$

where $m$ is mass of the ball, $d_{\mathrm{m}}^{\prime}$ is the pitch diameter under loading and $\omega_{c}$ is 
the angular velocity of the cage. The gyroscopic moment of the ball can be calculated as

$$
M_{\mathrm{g}}=I_{\mathrm{p}} \omega_{\mathrm{B}} \omega_{\mathrm{c}} \sin \theta
$$

where $I_{\mathrm{p}}$ is the mass moment of inertia of the ball, $\omega_{\mathrm{B}}$ is the angular velocity of the ball about its rotation axis, $\omega_{\mathrm{c}}$ is the angular velocity of the cage and $\theta$ is the angle between the shaft center line and ball rotational axis. Equation 17 can be re-expressed as

$$
\begin{aligned}
& \frac{K_{\mathrm{o}} \delta_{\mathrm{o}}^{3 / 2} L_{3}}{d\left(f_{\mathrm{o}}-0.5\right)+\delta_{\mathrm{o}}}-\frac{K_{\mathrm{i}} \delta_{\mathrm{i}}^{3 / 2}\left(D \sin \alpha_{\mathrm{f}}+\delta_{\mathrm{t}}-L_{3}\right)}{d\left(f_{\mathrm{i}}-0.5\right)+\delta_{\mathrm{i}}} \\
& -\frac{2}{d} I_{\mathrm{p}} \omega_{\mathrm{B}} \omega_{\mathrm{c}} \sin \theta\left(\frac{\lambda L_{4}}{d\left(f_{\mathrm{o}}-0.5\right)+\delta_{\mathrm{o}}}-\frac{(1-\lambda)\left(D \cos \alpha_{\mathrm{f}}+\delta_{\mathrm{r}} \cos \psi-L_{4}\right)}{d\left(f_{\mathrm{i}}-0.5\right)+\delta_{\mathrm{i}}}\right)=0 \\
& \frac{K_{\mathrm{o}} \delta_{\mathrm{o}}^{3 / 2} L_{4}}{d\left(f_{\mathrm{o}}-0.5\right)+\delta_{\mathrm{o}}}-\frac{K_{\mathrm{i}} \delta_{\mathrm{i}}^{3 / 2}\left(D \cos \alpha_{\mathrm{f}}+\delta_{\mathrm{r}} \cos \psi-L_{4}\right)}{d\left(f_{\mathrm{i}}-0.5\right)+\delta_{\mathrm{i}}} \\
& +\frac{2}{d} I_{\mathrm{p}} \omega_{\mathrm{B}} \omega_{\mathrm{c}} \sin \theta\left(\frac{\lambda L_{3}}{d\left(f_{\mathrm{o}}-0.5\right)+\delta_{\mathrm{i}}}-\frac{(1-\lambda)\left(D \sin \alpha_{\mathrm{f}}+\delta_{\mathrm{t}}-L_{3}\right)}{d\left(f_{\mathrm{i}}-0.5\right)+\delta_{\mathrm{i}}}\right) \\
& -\frac{m \omega_{\mathrm{c}}^{2}}{2}\left(d_{\mathrm{e}}+2 L_{4}-2\left(f_{\mathrm{o}}-0.5\right) d \cos \alpha_{\mathrm{f}}\right)=0 .
\end{aligned}
$$

The iterative solution is required due the nonlinear equations and the Newton-Raphson method is utilized to solve the four equations in Eq. 15 and Eq. 20. The unknown variables to solve are $L_{3}, L_{4}, \delta_{\mathrm{i}}$ and $\delta_{\mathrm{o}}$. Good assumptions for tangential and radial displacement, $\delta_{\mathrm{t}}$ and $\delta_{\mathrm{r}}$, can be made by using a condition of equilibrium applied to the complete ball bearing as follows

$$
\begin{aligned}
& F_{\mathrm{t}}-\sum_{\mathrm{k}=1}^{n}\left[F_{\mathrm{kj}} \sin \alpha_{\mathrm{kj}}-\frac{2\left(1-\lambda_{\mathrm{j}}\right) M_{\mathrm{gj}}}{d} \cos \alpha_{\mathrm{kj}}\right]=0, \\
& F_{\mathrm{r}}-\sum_{\mathrm{k}=1}^{n}\left[F_{\mathrm{kj}} \cos \alpha_{\mathrm{kj}}-\frac{2\left(1-\lambda_{\mathrm{j}}\right) M_{\mathrm{gj}}}{d} \sin \alpha_{\mathrm{kj}}\right] \cos \psi_{\mathrm{j}}=0,
\end{aligned}
$$


where $F_{\mathrm{t}}$ is the axial load and $F_{\mathrm{r}}$ is the radial load. [3]

\section{Case studies and results}

The two developed models are implemented into a three different sized rotors. In size order from largest to smallest the studied cases are a solid rotor blower (turbo blower), a high-speed machine and a high-speed generator. The turbo blower is supported with two 6014 hybrid deep-groove ball bearings, the machine with two $71911 \mathrm{CE} / \mathrm{HCP} 4 \mathrm{~A}$ high precision ball bearings and the generator with two S7005 CE/HCP4A high precision angular contact ball bearings. The turbo blower rotor's geometry is similar to that presented earlier in Pyrhönen et al. [18]. However, in this study, the constant bearing stiffnesses used in the earlier work have been replaced by stiffnesses obtained using the developed ball bearing models. The structure for the high-speed machine and high-speed generator are presented in the following sections.

In the cases inner race bearing stiffness coefficients, critical speed maps and Campbell diagrams are studied.

\section{1. a) Turbo blower structure}

The main layout and dimensions of the turbo blower are shown in Fig. 7 while the parameters used in the analysis are shown in Tab. 1. 


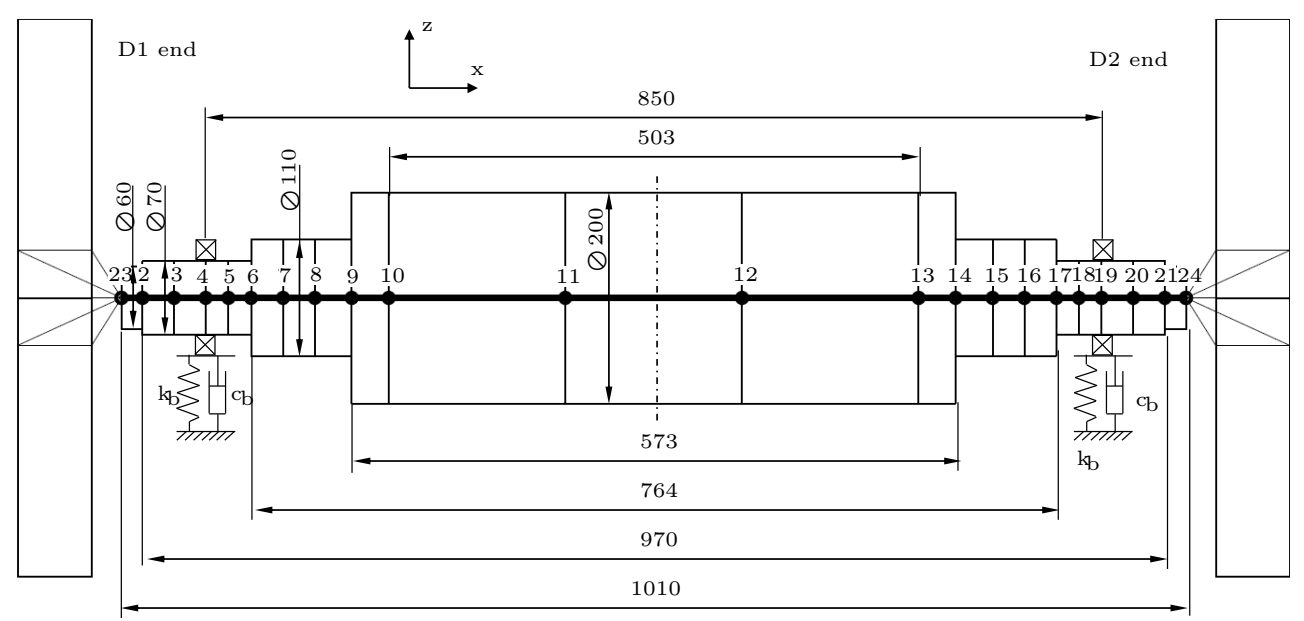

Figure 7: Layout and finite element discretization of the turbo blower with two impellers. The dimensions are in millimeters. The slitted part of the rotor is between nodes 10 and 13.

Table 1: Parameters used in the turbo blower analysis.

\begin{tabular}{ll}
\hline Young's modulus & $210 \mathrm{GPa}$ \\
Material density & $7800 \frac{\mathrm{kg}}{\mathrm{m}^{3}}$ \\
Poisson's ratio (rotor) & 0.3 \\
Properties of the slitted part & \\
Area & $0.0251 \mathrm{~m}^{2}$ \\
Second moment of area & $6.2832 \times 10^{-5} \mathrm{~m}^{4}$ \\
Impeller mass properties & \\
Mass & $19.0 \mathrm{~kg}$ \\
Polar mass moment of inertia & $0.5 \mathrm{~kg} \mathrm{~m}{ }^{2}$ \\
Diametral mass moment of inertia & $0.3 \mathrm{~kg} \mathrm{~m}{ }^{2}$ \\
Stiffness of impeller attachment & \\
Translational & $1.0 \times 10^{11} \frac{\mathrm{N}}{\mathrm{m}}$ \\
Rotational & $1.5 \times 10^{6} \frac{\mathrm{N} \mathrm{m}}{\mathrm{rad}}$ \\
Rotor unbalance masses & $36 \mathrm{~g} \mathrm{~mm} @ 270^{\circ}$ \\
D1 Impeller (node 23) & $50 \mathrm{~g} \mathrm{~mm} @ 0^{\circ}$ \\
D1 Rotor end (node 9) & $50 \mathrm{~g} \mathrm{~mm} @ 0^{\circ}$ \\
D2 Rotor end (node 14) & $50 \mathrm{~g} \mathrm{~mm} @ 90^{\circ}$ \\
D2 Impeller (node 24) & $174.85 \mathrm{~kg}$ \\
Total mass of the rotor & \\
\hline
\end{tabular}


The turbo blower is supported with two SKF 6014/HC2 deep-groove ball bearings and those are located in nodes 4 and 19 (Fig. 7). The main bearing dimension and parameters are shown in the Tab. 2.

Table 2: Dimensions of the 6014/HC type deep-groove hybrid ball bearing

\begin{tabular}{lll}
\hline Bore diameter & $d_{\mathrm{s}}$ & $70 \mathrm{~mm}$ \\
Outer diameter & $D$ & $110 \mathrm{~mm}$ \\
Width & $W$ & $20 \mathrm{~mm}$ \\
Pitch diameter & $d_{\mathrm{m}}$ & $89.85 \mathrm{~mm}$ \\
Ball diameter & $d$ & $11.91 \mathrm{~mm}$ \\
Number of balls & $n$ & 14 \\
Diametral clearance & $c_{\mathrm{d}}$ & $5.5 \mu \mathrm{m}$ \\
Bearing damping coefficient & $c_{\mathrm{b}}$ & $0.55 \frac{\mathrm{Ns}}{\mathrm{mm}}$ \\
Inner and outer race conformity & $R_{\mathrm{i}}, R_{\mathrm{O}}$ & 0.52 \\
Static load rating & $C_{0}$ & $31000 \mathrm{~N}$ \\
Modulus of elasticity (races) & $E_{\mathrm{r}}$ & $207 \mathrm{GPa}$ \\
Poisson's ratio (races) & $\nu_{\mathrm{r}}$ & 0.3 \\
Modulus of elasticity (balls) & $E_{\mathrm{b}}$ & $315 \mathrm{GPa}$ \\
Density (balls) & $\rho_{\mathrm{b}}$ & $3200 \frac{\mathrm{kg}}{\mathrm{m}^{3}}$ \\
Poisson's (balls) & $\nu_{\mathrm{b}}$ & 0.26 \\
Reference speed & $n_{\mathrm{max}}$ & $16.000 \mathrm{rpm}$ \\
Misalignment & $\gamma_{\mathrm{y}}$ & $0^{\circ}$ \\
\hline
\end{tabular}

Both bearings are loaded with a $300 \mathrm{~N}$ axial load and a $700 \mathrm{~N}$ radial load (z-direction). The bearing support is considered to be rigid. The initial guess for the radial displacement, $\delta_{r}$, is $70 \mu \mathrm{m}$ and for the axial direction, $\delta_{t}$, is $10 \mu \mathrm{m}$. Using Equations 15 and 20 the initial deformations $\delta_{i}, \delta_{o}$ and initial distances $L_{3}$ and $L_{4}$ can be obtained.

\section{2. b) High-speed machine structure}

The main layout and dimensions of the high-speed machine are shown in Fig. 8 while the parameters used in the analysis are shown in Tab. 3 . 


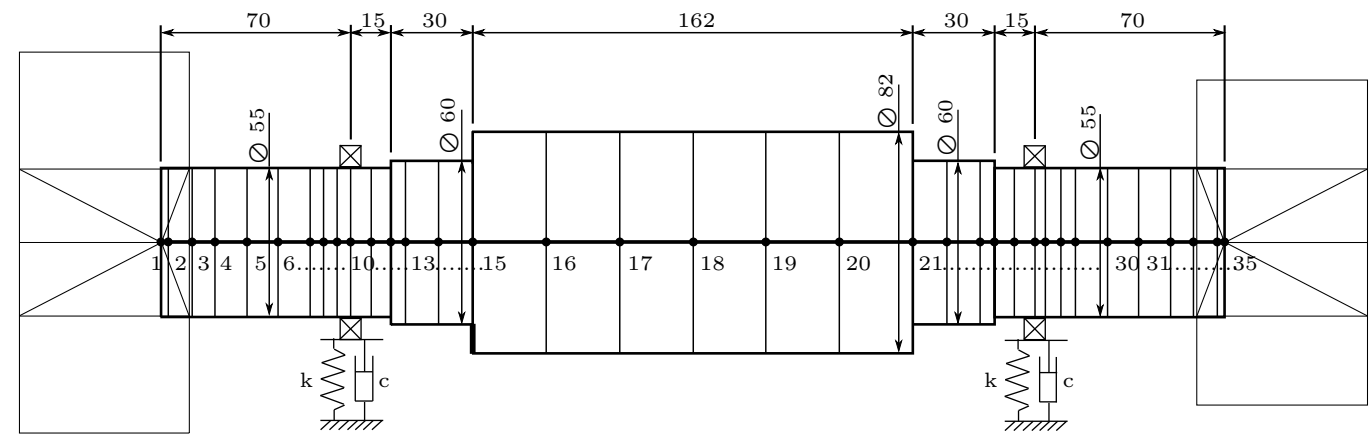

Figure 8: Layout and finite element discretization of the high-speed machine. The dimensions are in millimeters. The impellers are located at nodes 1 and 35 .

Table 3: High-speed machine parameters used in analysis

\begin{tabular}{ll}
\hline Young's modulus (rotor) & $210 \mathrm{GPa}$ \\
Material density (rotor) & $7800 \frac{\mathrm{kg}}{\mathrm{m}^{3}}$ \\
Poisson's ratio (rotor) & 0.3 \\
Impeller mass properties & $6.0 \mathrm{~kg}$ \\
Mass (node 1) & $0.055 \mathrm{~kg} \mathrm{~m}^{2}$ \\
Polar mass moment of inertia & $0.031 \mathrm{~kg} \mathrm{~m}^{2}$ \\
Diametral mass moment of inertia & $5.1 \mathrm{~kg}$ \\
Mass (node 35) & $0.028 \mathrm{~kg} \mathrm{~m}^{2}$ \\
Polar mass moment of inertia & $0.017 \mathrm{~kg} \mathrm{~m}{ }^{2}$ \\
Diametral mass moment of inertia & \\
Rotor unbalance masses & $0.681 \mathrm{~g} \mathrm{~mm} @ 0^{\circ}$ \\
Rotor (node 15 and 21) & $0.165 \mathrm{~g} \mathrm{~mm} @ 0^{\circ}$ \\
machine (node 1) & $0.135 \mathrm{~g} \mathrm{~mm} @ 0^{\circ}$ \\
machine (node 35) & $26.0 \mathrm{~kg}$ \\
\hline Total mass of the rotor &
\end{tabular}

The high-speed machine is supported with two $71911 \mathrm{CE} / \mathrm{HCP} 4 \mathrm{~A}$ angular contact ball bearings. The bearings are located at nodes 9 and 25 (Fig. 8). The main bearing dimension and parameters are shown in the Tab. 4. 
Table 4: Dimensions of the $71911 \mathrm{CE} / \mathrm{HCP} 4 \mathrm{~A}$ angular contact ball bearing

\begin{tabular}{lll}
\hline Bore diameter & $d_{\mathrm{s}}$ & $55 \mathrm{~mm}$ \\
Outer diameter & $D$ & $80 \mathrm{~mm}$ \\
Width & $W$ & $13 \mathrm{~mm}$ \\
Pitch diameter & $d_{\mathrm{m}}$ & $67.538 \mathrm{~mm}$ \\
Ball diameter & $d$ & $7.938 \mathrm{~mm}$ \\
Number of balls & $n$ & 21 \\
Diametral clearance & $c_{\mathrm{d}}$ & $5.5 \mu \mathrm{m}$ \\
Inner and outer race conformity & $R_{\mathrm{i}}, R_{\mathrm{O}}$ & 0.52 \\
Static load rating & $C_{0}$ & $10600 \mathrm{~N}$ \\
Modulus of elasticity (races) & $E_{\mathrm{r}}$ & $207 \mathrm{GPa}$ \\
Poisson's ratio (races) & $v_{\mathrm{r}}$ & 0.3 \\
Modulus of elasticity (balls) & $E_{\mathrm{b}}$ & $315 \mathrm{GPa}$ \\
Density (balls) & $\rho_{\mathrm{b}}$ & $3200 \frac{\mathrm{kg}}{\mathrm{m}^{3}}$ \\
Poisson's (balls) & $v_{\mathrm{b}}$ & 0.26 \\
Reference speed & $n_{\mathrm{max}}$ & $43000 \mathrm{rpm}$ \\
Misalignment & $\gamma_{\mathrm{y}}$ & $0^{\circ}$ \\
\hline
\end{tabular}

Both bearings are loaded with a $800 \mathrm{~N}$ axial load and a $90 \mathrm{~N}$ radial load (z-direction). The bearing support is considered to be rigid. The initial guess for the radial displacement, $\delta_{r}$, is $50 \mu \mathrm{m}$ and for the axial direction, $\delta_{t}$, is $10 \mu \mathrm{m}$. Using Equations 15 and 20 the initial deformations $\delta_{i}, \delta_{o}$ and initial distances $L_{3}$ and $L_{4}$ can be obtained.

\section{3. c) High-speed generator structure}

The main layout and dimensions of the high-speed generator are shown in Fig. 9 while the parameters used in the analysis are shown in Tab. 5. 


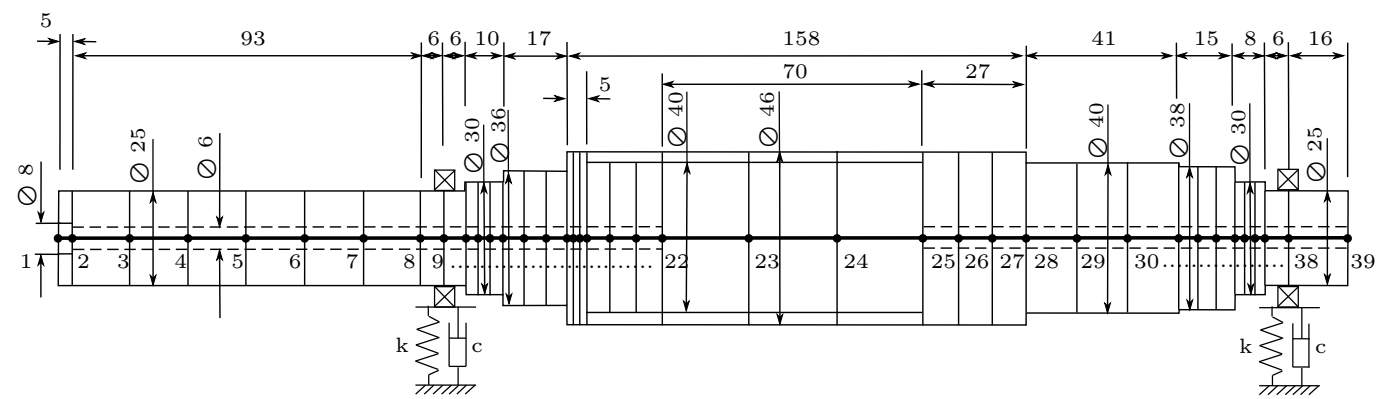

Figure 9: Layout and finite element discretization of the high-speed generator. A permanent magnet is located between nodes 22 and 25. The dimensions are in millimeters.

Table 5: Parameters used in the high-speed generator analysis

\begin{tabular}{ll} 
Young's modulus (rotor) & $210 \mathrm{GPa}$ \\
Material density (rotor) & $7800 \frac{\mathrm{kg}}{\mathrm{m}^{3}}$ \\
Poisson's ratio (rotor) & 0.3 \\
Young's modulus (PM) & $150 \mathrm{GPa}$ \\
Material density (PM) & $8400 \frac{\mathrm{kg}}{\mathrm{m}^{3}}$ \\
Poisson's ratio (PM) & 0.3 \\
Rotor unbalance masses & \\
Rotor end (node 1) & $0.485 \mathrm{~g} \mathrm{~mm} @ 0^{\circ}$ \\
Permanent Magnet (node 22) & $0.148 \mathrm{~g} \mathrm{~mm} @-90^{\circ}$ \\
Total mass of the rotor & $2.5 \mathrm{~kg}$ \\
\hline
\end{tabular}

The high-speed generator is supported with two S7005 CE/HCP4A angular contact ball bearing. The bearings are located at nodes 9 and 38 (Fig. 9). The main bearing dimension and parameters are shown in the Tab. 6 . 
Table 6: Dimensions of the S7005 CE/HCP4A angular contact ball bearing

\begin{tabular}{lll}
\hline Bore diameter & $d_{\mathrm{s}}$ & $25 \mathrm{~mm}$ \\
Outer diameter & $D$ & $47 \mathrm{~mm}$ \\
Width & $W$ & $12 \mathrm{~mm}$ \\
Pitch diameter & $d_{\mathrm{m}}$ & $35.15 \mathrm{~mm}$ \\
Ball diameter & $d$ & $6.35 \mathrm{~mm}$ \\
Number of balls & $n$ & 14 \\
Diametral clearance & $c_{\mathrm{d}}$ & $5.5 \mu \mathrm{m}$ \\
Inner and outer race conformity & $R_{\mathrm{i}}, R_{\mathrm{O}}$ & 0.52 \\
Static load rating & $C_{0}$ & $4150 \mathrm{~N}$ \\
Modulus of elasticity (races) & $E_{\mathrm{r}}$ & $207 \mathrm{GPa}$ \\
Poisson's ratio (races) & $v_{\mathrm{r}}$ & 0.3 \\
Modulus of elasticity (balls) & $E_{\mathrm{b}}$ & $315 \mathrm{GPa}$ \\
Density (balls) & $\rho_{\mathrm{b}}$ & $3200 \frac{\mathrm{kg}}{\mathrm{m}}$ \\
Poisson's (balls) & $v_{\mathrm{b}}$ & 0.26 \\
Reference speed & $n_{\mathrm{max}}$ & $56000 \mathrm{rpm}$ \\
Misalignment & $\gamma_{\mathrm{y}}$ & $0^{\circ}$ \\
\hline
\end{tabular}

Both bearings are loaded with a $265 \mathrm{~N}$ axial load and a $20 \mathrm{~N}$ radial load (z-direction). The bearing support is considered to be rigid. The initial guess for the radial displacement, $\delta_{r}$, is $50 \mu \mathrm{m}$ and for the axial direction, $\delta_{t}$, is $10 \mu \mathrm{m}$. Using Equations 15 and 20 the initial deformations $\delta_{i}, \delta_{o}$ and initial distances $L_{3}$ and $L_{4}$ can be obtained.

\subsection{Results of the simulations}

Two bearing models were implemented into three different size rotor models. An analysis was performed using a custom in-house code in Matlab software. The rotor is modeled by finite element method (FEM) [19] and the used element type is the Timoshenko beam element [20]. The stiffness of the bearing inner race, critical speed maps and Campbell diagrams are studied. 


\subsubsection{Ball bearing stiffness coefficients}

In the simplified ball bearing model, the bearing stiffness coefficient does not depend on the rotation speed. On the contrary, in a high-speed bearing model, the bearing stiffnesses are strongly dependent of the rotation speed. The diagonal stiffness values for the simplified bearing model in the translational direction are shown in the Tab 7.

\begin{tabular}{cccc} 
Table 7: Axial and radial stiffnesses with the simplified bearing model \\
\hline Model & Axial stiffness & Radial stiffness & Radial stiffness \\
& $x\left(\frac{\mathrm{N}}{\mathrm{m}}\right)$ & $y\left(\frac{\mathrm{N}}{\mathrm{m}}\right)$ & $z\left(\frac{\mathrm{N}}{\mathrm{m}}\right)$ \\
Turbo blower & $2.021 \times 10^{7}$ & $2.8445 \times 10^{8}$ & $2.7784 \times 10^{8}$ \\
High-speed machine & $5.628 \times 10^{7}$ & $4.0941 \times 10^{8}$ & $4.0958 \times 10^{8}$ \\
High-speed generator & $2.648 \times 10^{7}$ & $2.0158 \times 10^{8}$ & $2.0158 \times 10^{8}$ \\
\hline
\end{tabular}

In the turbo blower model, the bearing is designed for a maximum rotation speed of $16000 \mathrm{rpm}$ which was also kept as the maximum value for the stiffness calculation. The high-speed machine bearings were designed for a maximum of $43000 \mathrm{rpm}$ and the stiffness was studied to $45000 \mathrm{rpm}$. The smallest, high-speed generator bearings were designed for the maximum rotation speed of $56000 \mathrm{rpm}$. The generator bearing stiffness values were studied up to $70000 \mathrm{rpm}$. The stiffness values as a function of rotation speed with the improved bearing model are shown in Fig. 10 for the turbo blower (a), Fig. 11 for the high-speed machine (b) and Fig. 12 for the high-speed generator (c). In these figures, the stiffness calculated with the simplified bearing model is also included. 
(a)
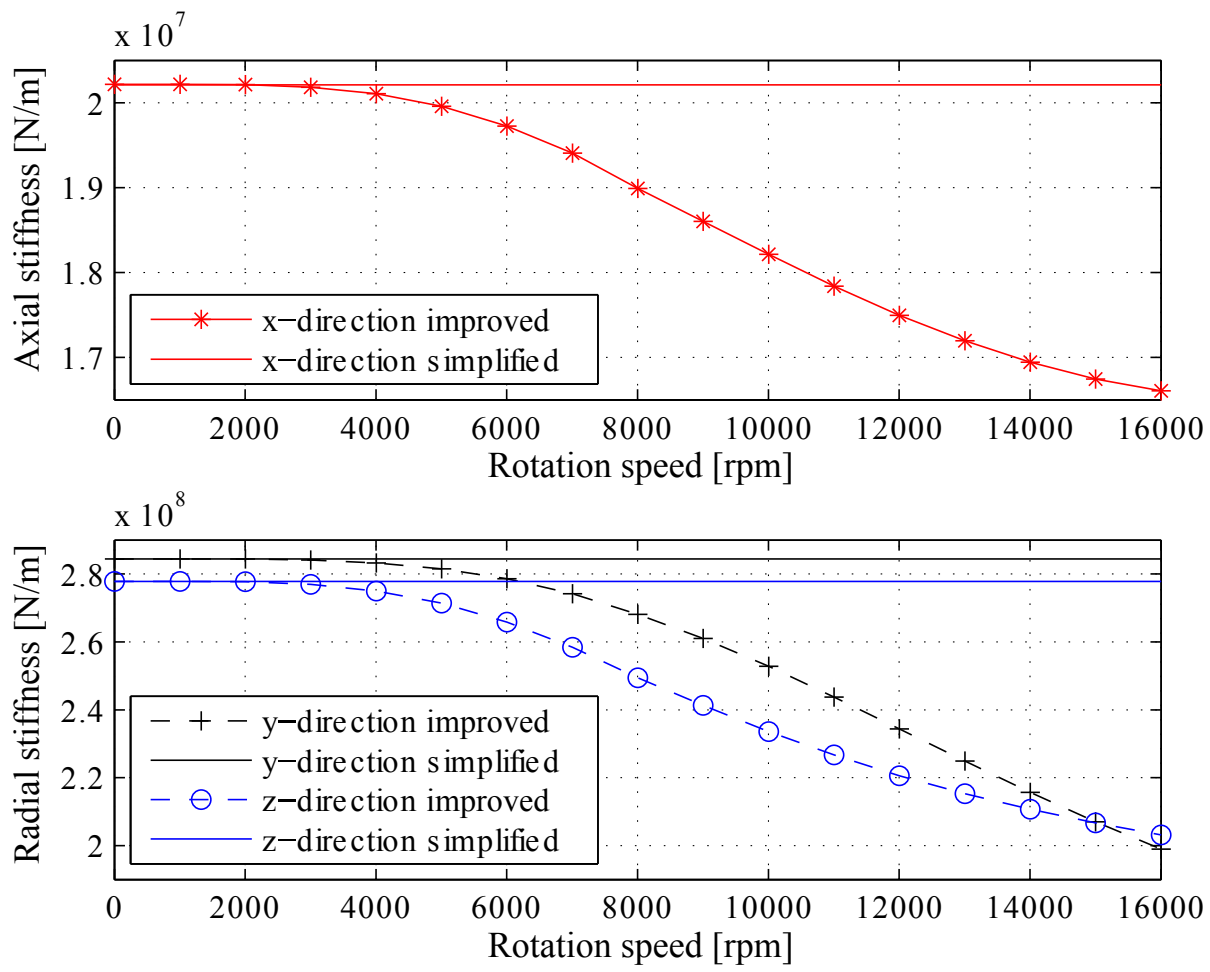

Figure 10: Bearing stiffnesses in the axial and radial directions as a function of rotation speed in turbo blower (0-16 $000 \mathrm{rpm})$

The stiffness coefficient in the blower decreases rapidly if the rotation speed is above $2000 \mathrm{rpm}$ and at the maximum rotation speed, the axial stiffness decreases by a total of $18.0 \%$, the radial direction in $y$-direction by $27.0 \%$ and the $z$-direction by $30.0 \%$ (i.e. direction of the applied force) from the initial stiffness coefficient. 
(b)
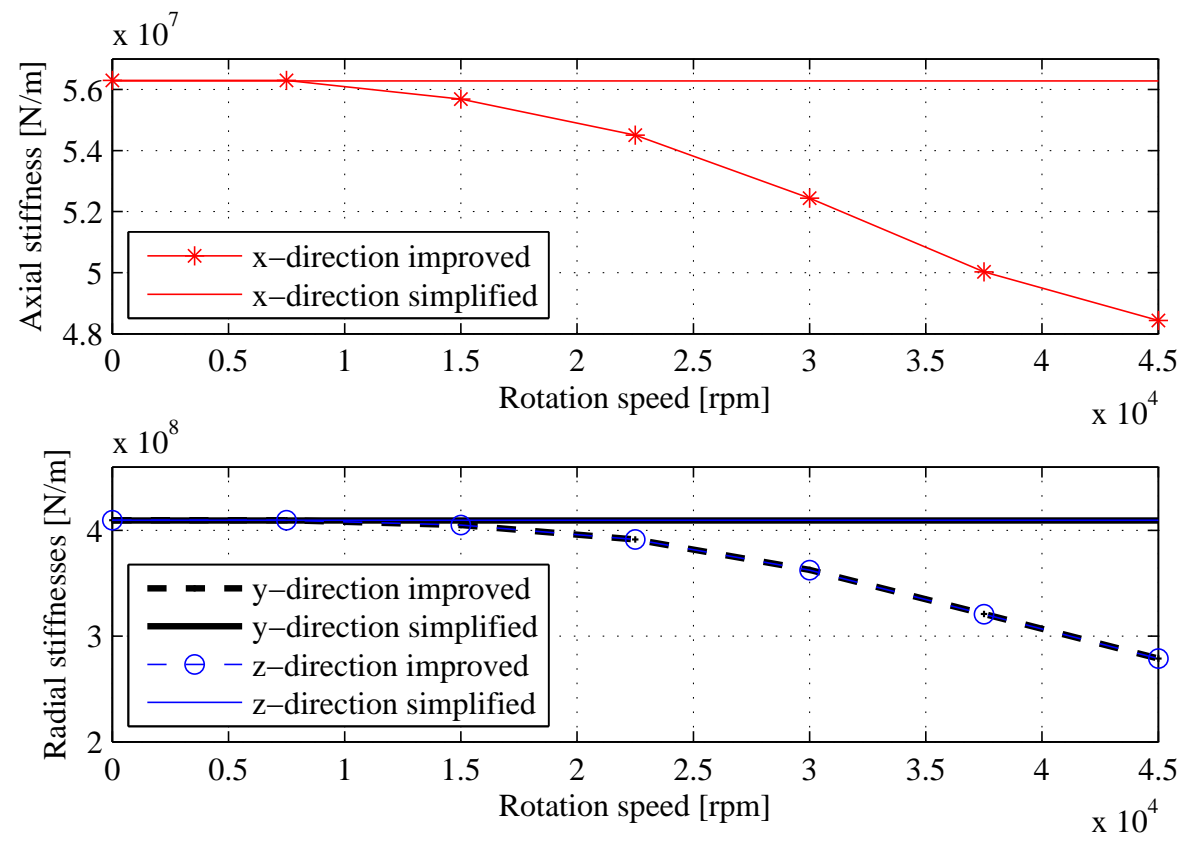

Figure 11: Bearing stiffnesses in the axial and radial directions as a function of rotation speed a high-speed machine (0-45000 rpm)

The stiffness coefficient in the high-speed machine decreases in the axial direction $14.0 \%$ and in the radial directions $31.9 \%$ from the original value to the value at $45000 \mathrm{rpm}$. 
(c)
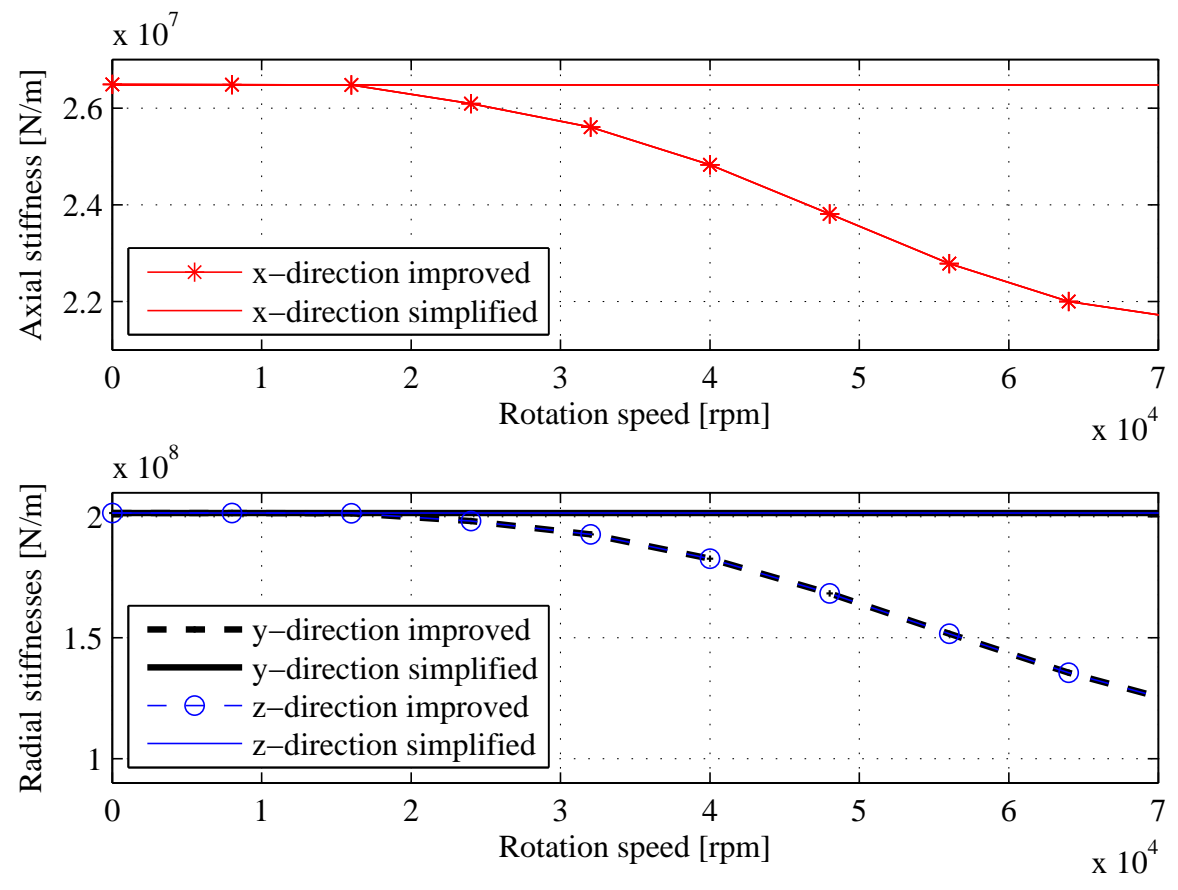

Figure 12: Bearing stiffnesses in the axial and radial directions as a function of the rotation speed of a high-speed generator (0-70000 rpm)

In the high-speed generator the axial stiffness decreases $18.3 \%$ and the radial stiffness $39.4 \%$ when comparing the stiffness value in the zero rotation speed and in the maximum calculated $70000 \mathrm{rpm}$.

In the studied cases, the stiffness decreases as the rotation speed increases and that is caused by the centrifugal force and gyroscopic moment affecting the ball. Based on Tab. 7 and Fig. 10, 11 and 12, it can be concluded that, at low rotation speeds, the stiffness coefficients are in good agreement between the two models. In the studied cases, when the stiffness begins to decrease a 
clear variation is visible. For the first case, a, when the rotor rotation speed is below $2000 \mathrm{rpm}$ the stiffness is constant. For the second case, b, when the rotor rotation speed is below $7500 \mathrm{rpm}$ the stiffness is constant. For the third case, c, when the rotor rotation speed is below $16000 \mathrm{rpm}$ the stiffness is constant. The rotation speeds and the velocity range when the stiffness is constant are shown in Tab. 8. The maximum rotation speed for each bearing is given by the manufacturer.

Table 8: Limiting rotation speeds when the stiffness starts to decrease

\begin{tabular}{lccc}
\hline Model & $\begin{array}{c}\text { Constant stiffness } \\
(\mathrm{rpm})\end{array}$ & $\begin{array}{c}\text { Bearing Maximum } \\
\text { rotation speed }(\mathrm{rpm})\end{array}$ & $\begin{array}{c}\text { Percentage from the } \\
\text { designed maximum }\end{array}$ \\
Turbo blower & $0-2000$ & 16000 & 12.5 \\
High-speed machine & $0-7500$ & 43000 & 17.4 \\
High-speed generator & $0-16000$ & 56000 & 28.6 \\
\hline
\end{tabular}

Based on these three cases, the range where the stiffness is constant shows a linear relationship with the bore diameter. The relationship shows that the smaller the bearing, the higher percentage from the maximum rotation speed the bearing can be rotated before the stiffness decreases due to the centrifugal force and gyroscopic moment affecting the balls.

\subsection{Critical speed maps}

In the critical speed map, the effect of support stiffness on the critical speeds are shown. From this, it can be seen how increasing the bearing stiffness affects the critical speeds. At low stiffness, the support stiffness (bearings) primarily defines the critical speeds. Once the support stiffness increases enough, the critical speeds reach a constant value (i.e. increasing the bearing stiffness has no effect on the critical speed). Above this point, 
the rotor flexibility defines the critical speeds. [21]

\section{a) Turbo blower}

In the critical speed map, the general relation between the support stiffness and the system can be seen. Figure 13 shows the critical speed map where the radial bearing stiffness ( $y$ - and $z$-directions) vary. The axial $(x)$ stiffness coefficient is set to zero. Critical speed maps are plotted on the zero rotational velocity for an unconstrained system. In the turbo blower, three first modes are shown.

a)

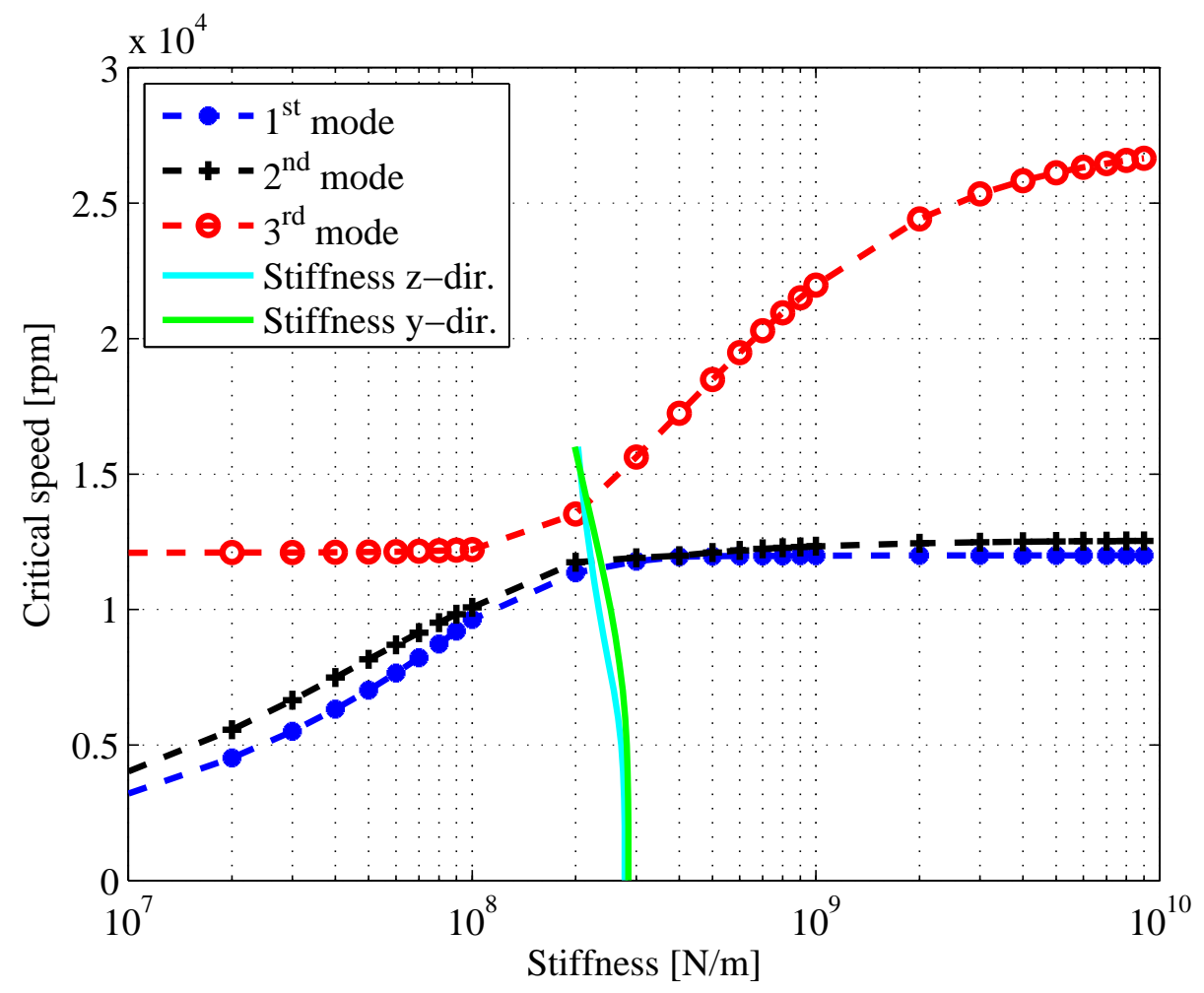

Figure 13: Undamped critical speed map. The radial stiffness is varied at zero velocity. 
The first two modes have an inclining section when the bearing stiffness increases above $2.0 \times 10^{8} \frac{\mathrm{N}}{\mathrm{m}}$. Below this value, the bearing stiffness mainly determines the critical speeds of the rotor system. Correspondingly, for bearing stiffnesses higher than $2.0 \times 10^{8} \frac{\mathrm{N}}{\mathrm{m}}$, the flexibility of the rotor has a more significant influence on the critical speeds. In this case, the variation of bearing stiffness will have an effect on the third mode but not on the first two modes.

\section{b) High-speed machine}

Figure 14 shows the critical speed map of the high-speed machine, where the radial bearing stiffness ( $y$ - and $z$-directions) varies and the two first modes are shown. 
b)

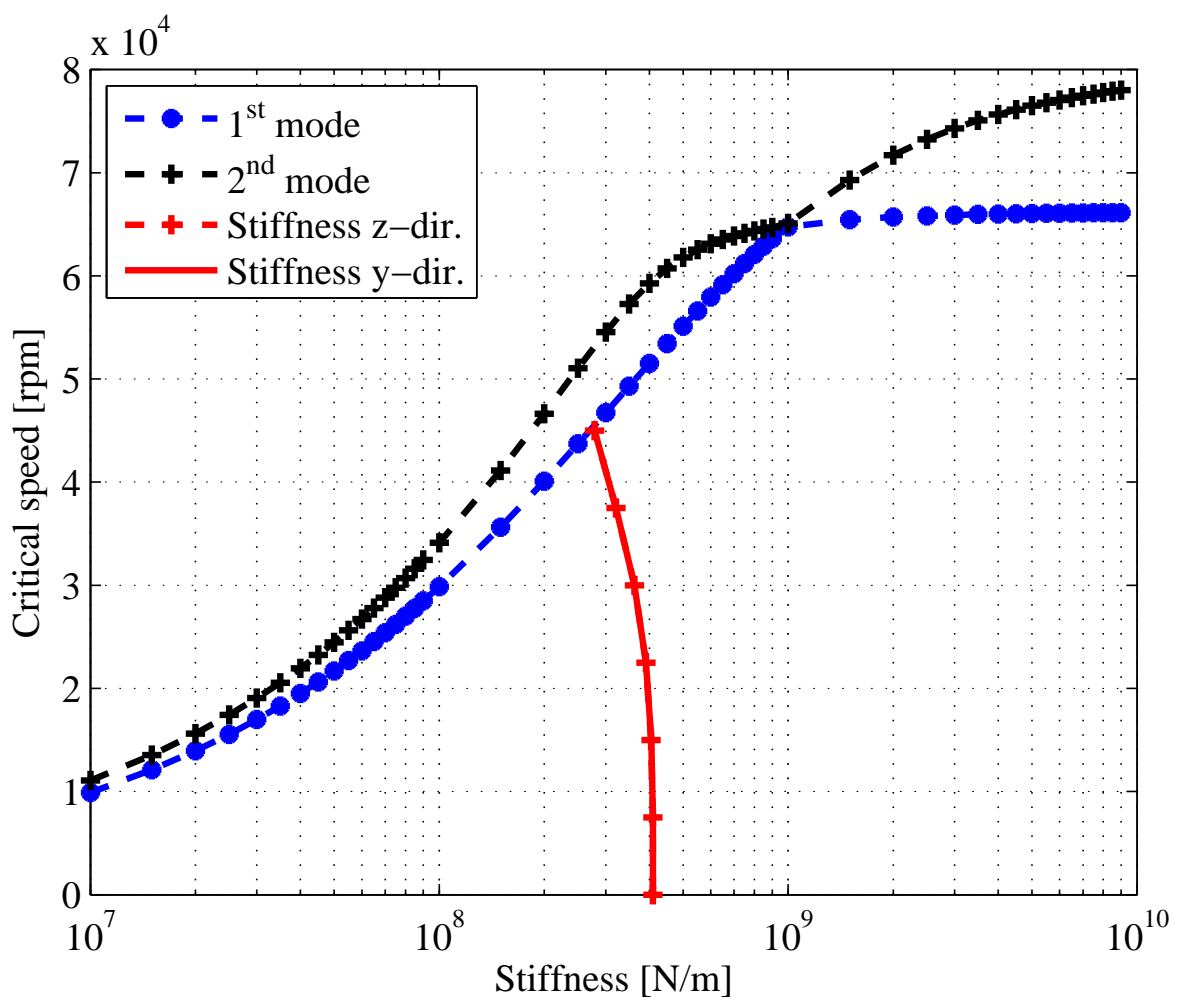

Figure 14: Critical speed map of high-speed machine

The two first modes have an inclining section when the bearing stiffness increases above $1.0 \times 10^{9} \frac{\mathrm{N}}{\mathrm{m}}$. Below this value, the bearing stiffness mainly determines the critical speed of the rotor system. Correspondingly, for bearing stiffnesses higher than $1.0 \times 10^{9} \frac{\mathrm{N}}{\mathrm{m}}$, the flexibility of the rotor has a more significant influence on the critical speeds. In this case, the bearing stiffness variation will have an effect on both modes.

c) High-speed generator 
Figure 15 shows the critical speed map of the high-speed generator, where the radial bearing stiffness ( $y$ - and $z$-directions) varies and the two first modes are shown.

c)

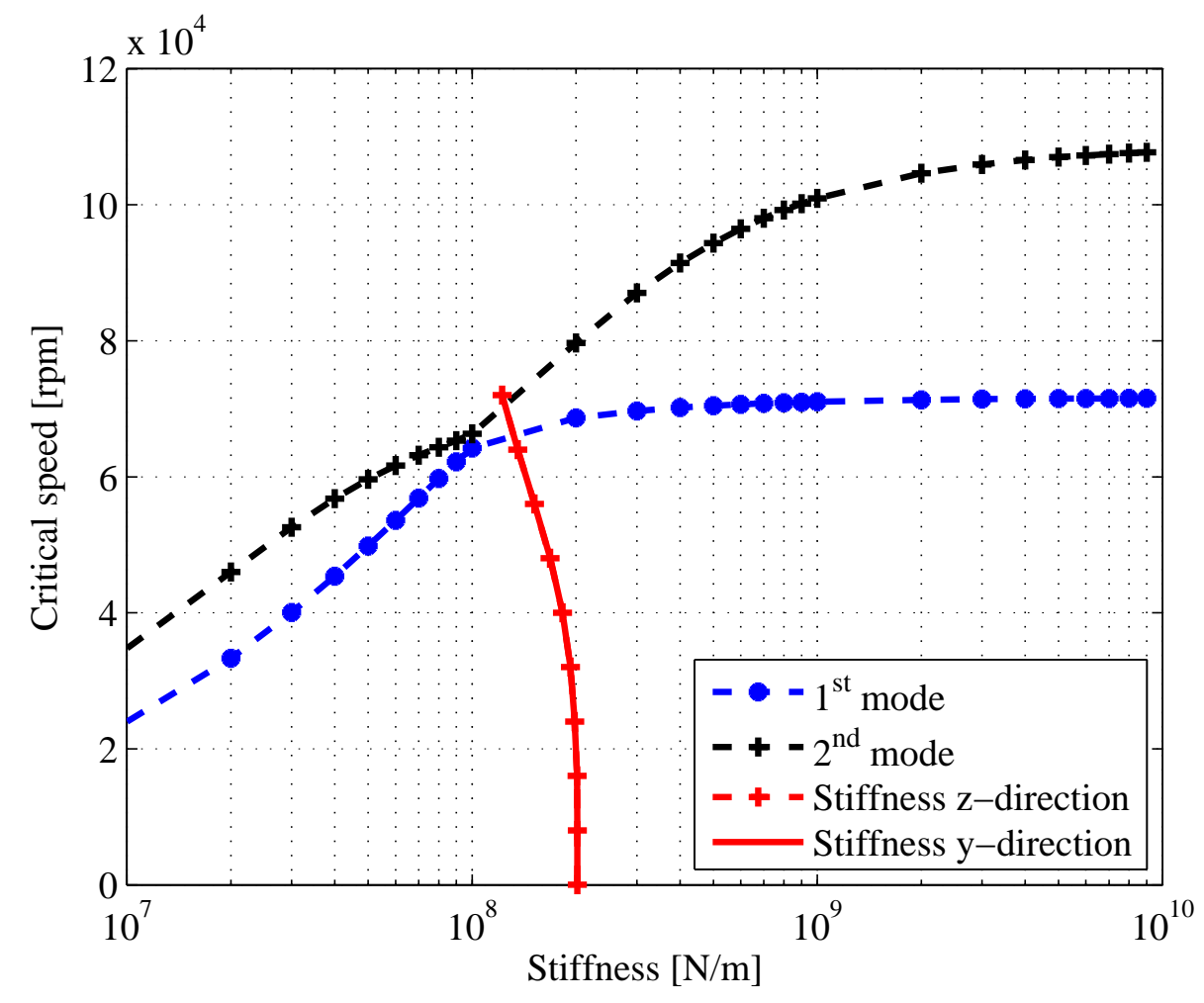

Figure 15: Critical speed map of high-speed generator

The two first modes have an inclining section when the bearing stiffness increases above $1.0 \times 10^{8} \frac{\mathrm{N}}{\mathrm{m}}$. Below this value, the bearing stiffness mainly determines the critical speed of the rotor system. Correspondingly, for bearing stiffnesses higher than $1.0 \times 10^{8} \frac{\mathrm{N}}{\mathrm{m}}$, the flexibility of the rotor has a more significant influence on the critical speeds. In this case, the variation of 
bearing stiffness will lower the critical speeds so that the second mode will decrease more than the first mode.

\subsubsection{Campbell diagrams}

a) Turbo blower

In a Campbell diagram, the natural frequencies of the rotor-bearing systems are shown as a function of rotation speed. The critical speeds are found from the diagram. The Campbell diagrams for the turbo blower with two bearing models are shown in Fig. 16. 
a)

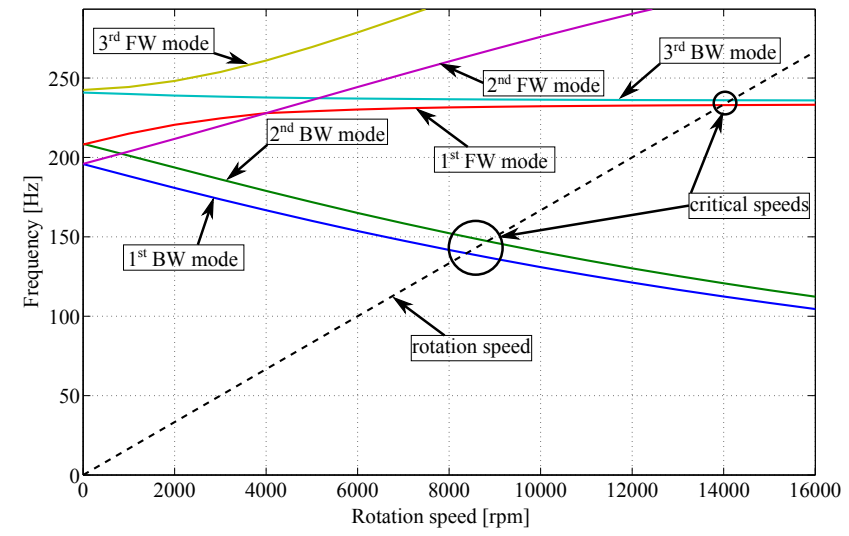

b)

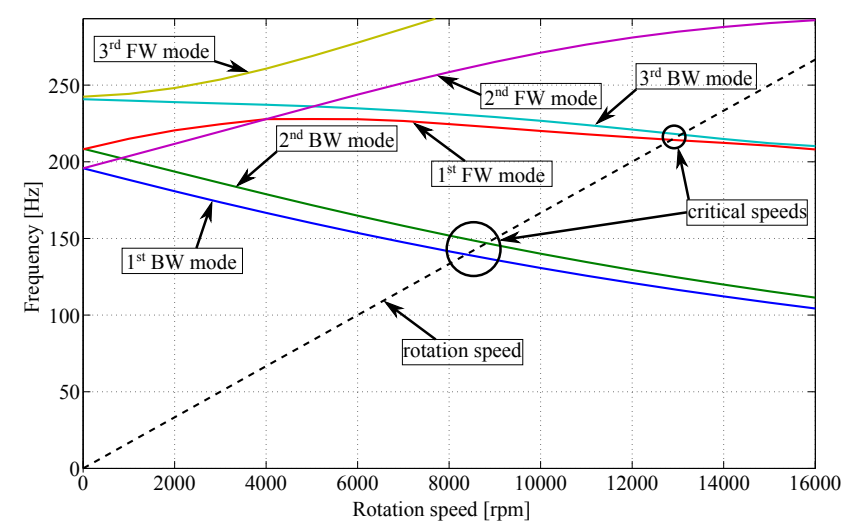

Figure 16: Campbell diagrams of the turbo blower: a) simplified bearing model and b) improved bearing model.

It can be seen in Fig. 16 that the Campbell diagrams are identical up to $6000 \mathrm{rpm}$ rotation speeds and as the rotation speed increases, the whirling frequencies start to differ. The first two critical speeds occur approximately at the same rotation speed in both bearing models. The third and fourth critical speeds occur in the improved bearing model at a lower rotation speed 
than in the simplified bearing model. The first critical speed is a backward whirling mode, the second is also a backward whirling mode, the third is a forward whirling mode and the fourth is a backward whirling mode (see Fig. 16. A comparison of the critical speeds in the two bearing models is shown in Tab. 9.

Table 9: Turbo blower critical rotation speed from the Campbell diagram

\begin{tabular}{llll}
\hline$\#$ & Critical speed rpm & & \\
& Simplified model & Improved model & Difference [\%] \\
1 & 8378 & 8372 & 0.07 \\
2 & 8836 & 8816 & 0.23 \\
3 & 13977 & 12861 & 8.68 \\
4 & 14160 & 13065 & 8.38 \\
\hline
\end{tabular}

The first two critical speeds occur close to each other and there is no clear difference between the models used to study this case. A clear difference can be seen at the third and fourth critical speeds. The critical speeds in the case of a high-speed ball bearing model are over $8 \%$ lower than with the simplified bearing model. The obtained results are in agreement with the critical speed map (Fig. 13) where the first and second critical speeds are not affected by the different bearing stiffnesses as the third and fourth critical are.

\section{b) High-speed machine}

The Campbell diagrams with the simplified and improved bearing models for the high-speed machine are shown in Fig. 17. 
a)

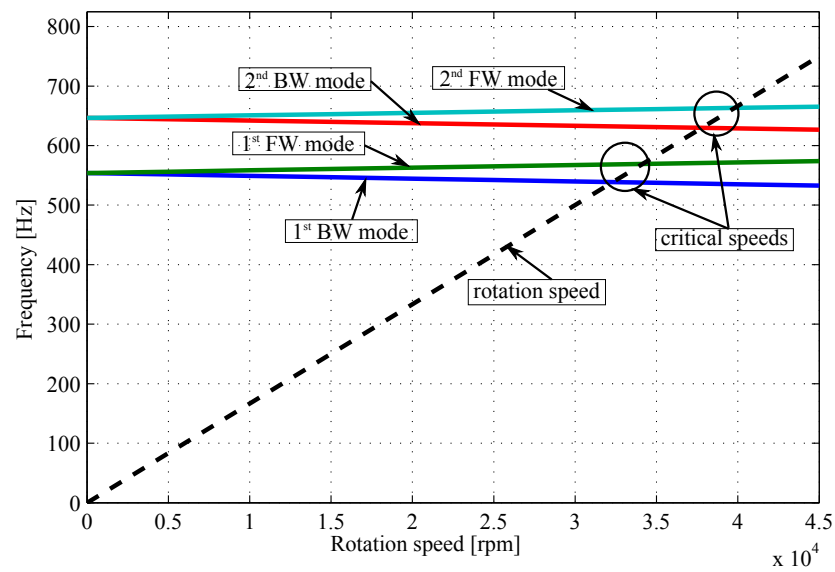

b)

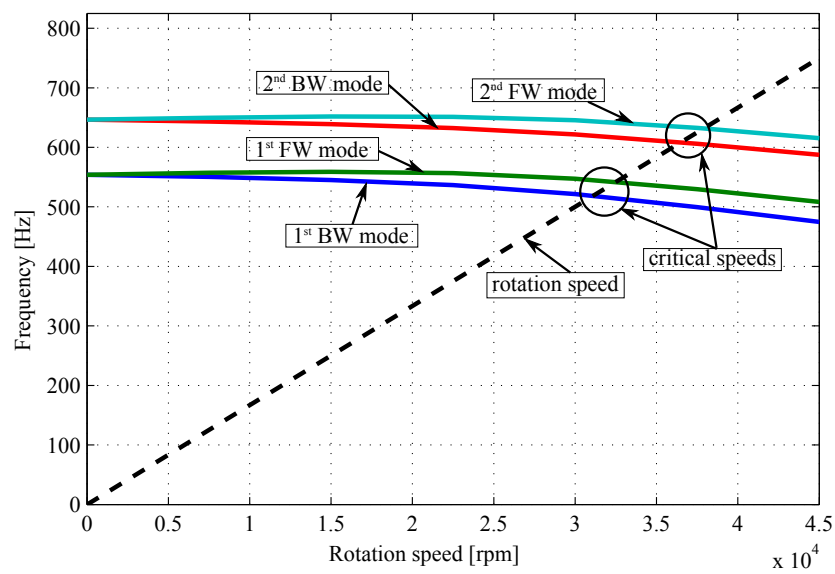

Figure 17: Campbell diagrams of the high-speed machine. a) simplified bearing model and b) improved bearing model.

It can be seen in Fig. 17 that the Campbell diagrams are in good agreement up to $15000 \mathrm{rpm}$ and as the rotation speed increases, the whirling frequencies start to differ. When the rotation speeds are faster than $15000 \mathrm{rpm}$, the whirling frequencies in the improved model are lower compared to the 
simplified model. With the high-speed machine, the differences in all the first four critical speeds are similar in magnitude. The faster the machine rotates, the bigger the difference between the two models are. The first critical speed is a backward whirling mode, the second is a forward whirling mode, the third is a backward whirling mode and the fourth is a forward whirling mode (see Fig. 17). A comparison of the critical speeds in the two bearing models is shown in Tab. 10.

Table 10: Critical rotations speed in high-speed machine from the Campbell diagram

\begin{tabular}{llll}
\hline$\#$ & Critical speed rpm & & \\
& Simplified model & Improved model & Difference [\%] \\
1 & 32324 & 31093 & 3.80 \\
2 & 34155 & 32480 & 4.90 \\
3 & 37796 & 36483 & 3.50 \\
4 & 39798 & 37910 & 4.70 \\
\hline
\end{tabular}

The critical speeds in the improved ball bearing model are from $3 \%$ to almost $5 \%$ lower than with the simplified bearing model. The difference is the same magnitude with all the first four critical speeds. It can also be seen from the critical speed map (Fig. 14) why the four first critical speeds have approximately a similar effect on the bearing stiffness.

\section{c) High-speed generator}

The Campbell diagrams for the high-speed generator are shown in Fig. 18. The Campbell diagram with the simplified model is calculated up to $90000 \mathrm{rpm}$ in order to show the third and fourth critical speeds. 
a)

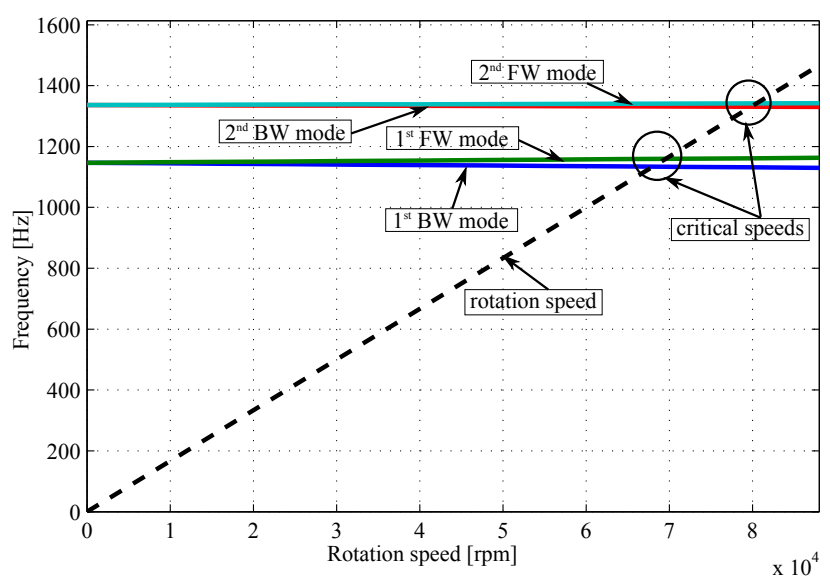

b)

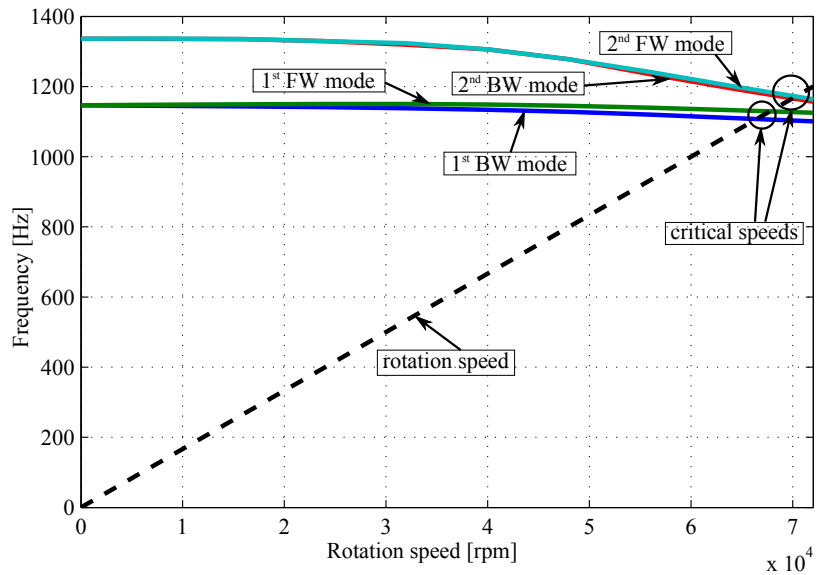

Figure 18: Campbell diagrams of the high-speed generator: a) simplified bearing model and b) improved bearing model.

It can be seen in Fig. 18 that at rotation speeds up to $16000 \mathrm{rpm}$, the Campbell diagrams are identical, and as the rotation speed increases, the whirling frequencies start to differ. The first two critical speeds occur approximately at the same rotation speed and the third and fourth critical 
speeds occurs at a lower rotation speed than in the simplified bearing model. The first critical speed is a backward whirling mode, the second is a forward whirling mode, the third is a backward whirling mode and the fourth is a forward whirling mode. A comparison of the critical speeds in the two models is shown in Tab. 11

Table 11: Critical rotations speed from the Campbell

\begin{tabular}{llll}
\multicolumn{2}{l}{ diagram } \\
\hline$\#$ & Critical speed rpm & \\
& Simplified model & Improved model & Difference [\%] \\
1 & 68026 & 66438 & 2.33 \\
2 & 69568 & 67756 & 2.60 \\
3 & $79853^{1}$ & 69981 & 12.36 \\
4 & $80501^{1}$ & 70300 & 12.67 \\
\hline \multicolumn{2}{l}{ occurs over the speed range }
\end{tabular}

The two first critical speeds occur close to each other and there is over $2 \%$ difference between the models used to study this case. A clearer difference can be seen in the third and fourth critical speeds. The critical speeds in the case of a high-speed ball bearing model are over $12 \%$ lower than with the simplified bearing model. The critical speed map (Fig. 15) clearly explains why the first and second critical speeds are not affected by the different bearing stiffnesses as the third and fourth critical are.

\section{Discussion}

In the case of ball bearings, an increase in model complexity leads to an increased number of input parameters and initial guesses that are crucial for successful simulation. The theoretical background for modelling ball bearings, including the centrifugal and gyroscopic forces, has been available since 
1960. Nevertheless, it is still unclear under which conditions (e.g. size of the rotor or rotation speed range) there is an obligation to use or not use the model including the high speed effects.

The studied cases show that the bearing stiffness coefficients are in agreement with the simplified and improved model at low rotation speeds. In fact, the results shows that in the improved bearing model, the bearings coefficients are almost constant up to a certain rotation speed. When the stiffness is constant, then there are no real benefits of using the improved ball bearing model. The largest case with the biggest bearing bore diameter, the turbo blower, concludes that it can be rotated up to $2000 \mathrm{rpm}$ with the simplified bearing model having zero difference to the improved model. The second largest case with the medium-sized bore diameter, the high-speed machine, concludes that it can be rotated up to $7500 \mathrm{rpm}$ having zero difference to the improved model. The smallest case, the high-speed generator, with the smallest bore diameter concludes that it can be rotated up to $16000 \mathrm{rpm}$ having zero difference to the improved model. It should be noted that in ball bearing modeling, there are several parameters and factors to be considered and the relationship shown in this paper is only valid with the studied cases.

In the literature, it is stated that for low and moderate speeds that the centrifugal and gyroscopic forces can be ignored and at high speeds, it is obligatory to consider them. For the studied rotors and with the used bearing types, the results are in agreement with the general statement in the literature. It should be noted that the results might vary even with the same structure when, for example, the clearance or bearing loading is varied. Due to this, common rules for the studied structures cannot be suggested. 
In all the cases the stiffness decreased when the rotation speed increased. Similar results are shown in the study by Butner et al. [22].

The critical speed map shows that the stiffness variation in the high speed bearing model does not decrease the stiffness sufficiently for it to have an influence on the first two critical speeds. The difference between the third and fourth critical speeds can also be seen in the critical speed map. As the bearing stiffness decreases, the critical speeds also decrease.

In the Campbell diagram, in the improved bearing model, the critical frequencies decreases as the rotation speed increases. The difference in the Campbell diagram calculations shows behavior similar to that which was presented in an early study by Cao and Altintas [23]. From the critical speed map at zero velocity, similar behavior can also be seen. Depending on which location the bearing stiffness is in on the critical speed map, (i.e. are the bearing stiffness defining the critical speeds or the rotor itself), the decrease in the critical speed varies in the studied cases.

The appropriate bearing model selection can be a subject of argument. The stiffness values for the studied rotors from both models (Figures 10, 11 and 12) show that if the values obtained with the improved model are close to the values obtained from the simplified model then the simplified model is justified. As the stiffness values diverge from the simplified model results when the speed is increased then the critical speed map should be plotted. A sensitivity analysis of the rotor critical speeds due to bearing stiffness variation should then be investigated. If the critical speeds are sensitive then the improved model should be used and the Campbell diagram should be plotted to see the detailed effects. Then the safety margin from the critical 
speeds to the specific operation speed can be determined.

\section{Conclusion}

The paper presented the implementation of two different ball bearing models in three rotors supported by two identical ball bearings, a turbo blower, a high-speed machine and a high-speed generator, respectively. The main findings from the study can be concluded as follows.

1. The ball bearing stiffness coefficients obtained from the two ball bearing models are identical in the turbo blower up to $2000 \mathrm{rpm}$, in the highspeed machine up to $7500 \mathrm{rpm}$ and in the high-speed generator up to $16000 \mathrm{rpm}$.

2. With the improved model, where the centrifugal force and gyroscopic moment is included, the stiffness for the turbo blower at $16000 \mathrm{rpm}$ is decreased by $18 \%$ in the axial direction, $27 \%$ in the radial direction $y$ and $30 \%$ in the radial direction $z$ where a load is applied. For the high-speed machine the axial stiffness is decreased at $45000 \mathrm{rpm} 14 \%$ in the axial direction, and $32 \%$ in the radial directions. For the highspeed generator at $70000 \mathrm{rpm}$ the stiffness is decreased $18 \%$ in the axial direction and $39 \%$ in the radial directions.

3. In the turbo blower case, a clear difference in the Campbell diagram is visible over $9000 \mathrm{rpm}$ between the simplified and improved bearing models. For this structure after $9000 \mathrm{rpm}$, the improved bearing model is justified. In the high-speed machine the two bearing models start to differ noticeably above $15000 \mathrm{rpm}$ and in the higher rotation speeds the improved model is justified. In the high-speed generator, the two 
bearing models differ at higher than $16000 \mathrm{rpm}$ then, the improved model is justified.

For future development, the studied models could be tested with actual bearings and could also try to eliminate parameters in order to propose an analytical equation for the modeling purpose of what the limiting rotation speed is when the improved model should be used.

\section{References}

[1] A. Jones, A General Theory for Elastically Constrained Ball and Radial Roller Bearings Under Arbitrary Load and Speed Conditions, Journal of Basic Engineering 82 (2) (1960) 309-320.

[2] T. A. Harris, M. N. Kotzalas, Advanced Concepts of Bearing Technology: Rolling Bearing Analysis, CRC Press, fifth edn., 2006.

[3] B. J. Hamrock, D. Dowson, Ball Bearing Lubrication: the Elastohydrodynamics of Elliptical Contacts, Wiley-Interscience, 1981.

[4] N. Tandon, A. Choudhury, A Review of Vibration and Acoustic Measurement Methods for the Detection of Defects in Rolling Element Bearings, Tribology International 32 (8) (1999) 469-480.

[5] N. Aktürk, M. Uneeb, R. Gohar, The Effects of Number of Balls and Preload on Vibrations Associated with Ball Bearings, Journal of Tribology 119 (4) (1997) 747-753.

[6] N. Aktürk, The Effect of Waviness on Vibrations Associated With Ball Bearings, Journal of Tribology 121 (4) (1999) 667-677. 
[7] G. Jang, S.-W. Jeong, Nonlinear Excitation Model of Ball Bearing Waviness in a Rigid Rotor Supported by Two or More Ball Bearings Considering Five Degrees of Freedom, Transactions-American Society of Mechanical Engineers Journal of Tribology 124 (1) (2002) 82-90.

[8] G. Jang, S.-W. Jeong, Analysis of a Ball Bearing with Waviness Considering the Centrifugal Force and Gyroscopic Moment of the Ball, Transactions-American Society of Mechanical Engineers Journal of Tribology 125 (3) (2003) 487-498.

[9] G. Jang, S.-W. Jeong, Vibration Analysis of a Rotating System Due to the Effect of Ball Bearing Waviness, Journal of Sound and Vibration 269 (3) (2004) 709-726.

[10] J. Sopanen, A. Mikkola, Dynamic Model of a Deep-Groove Ball Bearing Including Localized and Distributed Defects. Part 1: Theory, Proceedings of the Institution of Mechanical Engineers, Part K: Journal of Multi-body Dynamics 217 (3) (2003) 201-211.

[11] J. Sopanen, A. Mikkola, Dynamic Model of a Deep-Groove Ball Bearing Including Localized and Distributed Defects. Part 2: Implementation and Results, Proceedings of the Institution of Mechanical Engineers, Part K: Journal of Multi-body Dynamics 217 (3) (2003) 213-223.

[12] B. Changqing, X. Qingyu, Dynamic Model of Ball Bearings with Internal Clearance and Waviness, Journal of Sound and Vibration 294 (1) (2006) $23-48$. 
[13] C. Bai, H. Zhang, Q. Xu, Effects of Axial Preload of Ball Bearing on the nonlinear Dynamic Characteristics of a Rotor-Bearing System, Nonlinear Dynamics 53 (3) (2008) 173-190.

[14] M. Nakhaeinejad, M. D. Bryant, Dynamic Modeling of Rolling Element Bearings with Surface Contact Defects Using Bond Graphs, Journal of Tribology 133 (1) (2011) 1-12.

[15] L.-X. Xu, Y.-H. Yang, Y.-G. Li, C.-N. Li, S.-Y. Wang, Modeling and Analysis of Planar Multibody Systems Containing Deep Groove Ball Bearing with Clearance, Mechanism and Machine Theory 56 (1) (2012) 69-88.

[16] A. Liew, N. Feng, E. Hahn, Transient Rotordynamic Modeling of Rolling Element Bearing Systems, Journal of Engineering for Gas Turbines and Power 124 (4) (2002) 984-991.

[17] E. Kurvinen, J. Sopanen, A. Mikkkola, Comparison of Ball Bearing Model Performance with and without Centrifugal and Gyroscopic Forces, in: ASME International Mechanical Engineering Congress and Exposition, Proceedings (IMECE), ASME, 1-10, 2014.

[18] J. Pyrhönen, J. Nerg, A. Mikkola, J. Sopanen, T. Aho, Electromagnetic and Mechanical Design Aspects of a High-Speed Solid-Rotor Induction Machine with No Separate Copper Electric Circuit in the Megawatt Range, Electrical Engineering 91 (1) (2009) 35-49.

[19] G. Genta, Dynamics of Rotating Systems, Springer, 2005. 
[20] ANSYS, Inc. theory, SAS IP Press, twelfth edn., 2001.

[21] W. Chen, E. Gunter, Introduction to Dynamics of Rotor-bearing Systems, Trafford on Demand Pub, 2005.

[22] M. Butner, B. Murphy, R. Akian, The Influence of Mounting Compliance and Operating Conditions on the Radial Stiffness of Ball Bearings: Analytic and Test Results, ASME Rotatating Machinery and Vehicle Dynamics, DE 35 (1991) 155-162.

[23] Y. Cao, Y. Altintas, A General Method for the Modeling of SpindleBearing Systems, Journal of Mechanical Design 126 (6) (2004) 10891104. 